\title{
Updated fit to three neutrino mixing: status of leptonic CP violation
}

\author{
M.C. Gonzalez-Garcia, ${ }^{a, b}$ Michele Maltoni $^{c}$ and Thomas Schwetz ${ }^{d}$ \\ ${ }^{a}$ Institució Catalana de Recerca i Estudis Avançats (ICREA), \\ Departament d'Estructura $i$ Constituents de la Matèria and \\ Institut de Ciencies del Cosmos, Universitat de Barcelona, \\ Diagonal 647, E-08028 Barcelona, Spain \\ ${ }^{b}$ C.N. Yang Institute for Theoretical Physics, State University of New York at Stony Brook, \\ Stony Brook, NY 11794-3840, U.S.A. \\ ${ }^{c}$ Instituto de Física Teórica UAM/CSIC, Universidad Autónoma de Madrid, \\ Calle de Nicolás Cabrera 13-15, Cantoblanco, E-28049 Madrid, Spain \\ ${ }^{d}$ Oskar Klein Centre for Cosmoparticle Physics, Department of Physics, Stockholm University, \\ SE-10691 Stockholm, Sweden \\ E-mail: maria.gonzalez-garcia@stonybrook.edu, \\ michele.maltoni@csic.es, schwetz@fysik.su.se
}

ABSTRACT: We present a global analysis of solar, atmospheric, reactor and accelerator neutrino data in the framework of three-neutrino oscillations based on data available in summer 2014. We provide the allowed ranges of the six oscillation parameters and show that their determination is stable with respect to uncertainties related to reactor neutrino and solar neutrino flux predictions. We find that the maximal possible value of the Jarlskog invariant in the lepton sector is $0.033 \pm 0.010( \pm 0.027)$ at the $1 \sigma(3 \sigma)$ level and we use leptonic unitarity triangles to illustrate the ability of global oscillation data to obtain information on $\mathrm{CP}$ violation. We discuss "tendencies and tensions" of the global fit related to the octant of $\theta_{23}$ as well as the $\mathrm{CP}$ violating phase $\delta_{\mathrm{CP}}$. The favored values of $\delta_{\mathrm{CP}}$ are around $3 \pi / 2$ while values around $\pi / 2$ are disfavored at about $\Delta \chi^{2} \simeq 6$. We comment on the non-trivial task to assign a confidence level to this $\Delta \chi^{2}$ value by performing a Monte Carlo study of T2K data.

Keywords: Neutrino Physics, Solar and Atmospheric Neutrinos

ARXIV EPRINT: 1409.5439 


\section{Contents}

1 Introduction 1

2 Oscillation parameters: results of the global analysis 2

2.1 Data included in our analysis 2

2.2 Description of the results 3

3 Mixing matrix and leptonic CP violation $\quad 6$

4 Tension and tendencies $\quad 10$

$\begin{array}{lll}4.1 \text { Impact of reactor flux uncertainties } & 10\end{array}$

4.2 Determination of $\Delta m_{21}^{2}$ : solar and KamLAND 11

4.3 Determination of $\Delta m_{3 \ell}^{2}: \nu_{\mu}$ and $\nu_{e}$ disappearance 13

4.4 Mass ordering, $\theta_{23}$ octant and CP phase: role of different data sets 14

$\begin{array}{lll}4.5 & \text { Remarks on confidence levels for } \delta_{\mathrm{CP}} & 17\end{array}$

$\begin{array}{lll}5 & \text { Summary } & 20\end{array}$

$\begin{array}{ll}\text { A List of data used in the analysis } & 21\end{array}$

\section{Introduction}

Thanks to remarkable discoveries by a number of neutrino oscillation experiments it is now an established fact that neutrinos have mass and leptonic flavors are not symmetries of Nature [1, 2], see ref. [3] for an overview. Ignoring controversial indications for the existence of neutrino mass states at the $\mathrm{eV}$ scale (see ref. [4] and references therein) a consistent description of global data on neutrino oscillations is possible by assuming mixing among the three known neutrinos $\left(\nu_{e}, \nu_{\mu}, \nu_{\tau}\right)$, which can be expressed as quantum superpositions of three massive states $\nu_{i}(i=1,2,3)$ with masses $m_{i}$. This implies the presence of a leptonic mixing matrix in the weak charged current interactions $[5,6]$ which can be parametrized as [7]:

$$
U=\left(\begin{array}{ccc}
c_{12} c_{13} & s_{12} c_{13} & s_{13} e^{-i \delta_{\mathrm{CP}}} \\
-s_{12} c_{23}-c_{12} s_{13} s_{23} e^{i \delta_{\mathrm{CP}}} & c_{12} c_{23}-s_{12} s_{13} s_{23} e^{i \delta_{\mathrm{CP}}} & c_{13} s_{23} \\
s_{12} s_{23}-c_{12} s_{13} c_{23} e^{i \delta_{\mathrm{CP}}} & -c_{12} s_{23}-s_{12} s_{13} c_{23} e^{i \delta_{\mathrm{CP}}} & c_{13} c_{23}
\end{array}\right),
$$

where $c_{i j} \equiv \cos \theta_{i j}$ and $s_{i j} \equiv \sin \theta_{i j}$. In addition to the Dirac-type phase $\delta_{\mathrm{CP}}$, analogous to that of the quark sector, there may also be two physical phases associated to a possible Majorana character of neutrinos, which however are not relevant for neutrino oscillations $[8,9]$ and are therefore omitted in the present work. Given the observed hierarchy between the 
solar and atmospheric mass-squared splittings there are two possible non-equivalent orderings for the mass eigenvalues, which are conventionally chosen as

$$
\begin{aligned}
& \Delta m_{21}^{2} \ll\left(\Delta m_{32}^{2} \simeq \Delta m_{31}^{2}>0\right) ; \\
& \Delta m_{21}^{2} \ll-\left(\Delta m_{31}^{2} \simeq \Delta m_{32}^{2}<0\right),
\end{aligned}
$$

with $\Delta m_{i j}^{2} \equiv m_{i}^{2}-m_{j}^{2}$. As it is customary we refer to the first option, eq. (1.2), as Normal Ordering (NO), and to the second one, eq. (1.3), as Inverted Ordering (IO); in this form they correspond to the two possible choices of the sign of $\Delta m_{31}^{2}$. In this convention the angles $\theta_{i j}$ can be taken without loss of generality to lie in the first quadrant, $\theta_{i j} \in[0, \pi / 2]$, and the CP phase $\delta_{\mathrm{CP}} \in[0,2 \pi]$. In the following we adopt the (arbitrary) convention of reporting results for $\Delta m_{31}^{2}$ for NO and $\Delta m_{32}^{2}$ for IO, i.e., we always use the one which has the larger absolute value. Sometimes we will generically denote such quantity as $\Delta m_{3 \ell}^{2}$, with $\ell=1$ for $\mathrm{NO}$ and $\ell=2$ for IO.

In this article, we present an up-to-date (as of summer 2014) global analysis of solar, atmospheric, reactor and accelerator neutrino data in the framework of three-neutrino oscillations. Alternative recent global fits have been presented in refs. [10, 11]. In section 2 we describe the data used in our analysis (listed also in appendix A) and we present the results of the global analysis and the allowed ranges of the oscillation parameters. In section 3 we focus on our knowledge on CP violation, discussing the present status of the leptonic Jarlskog invariant and displaying the results of our fit in terms of leptonic unitarity triangles. In section 4 we comment on various "tensions and tendencies" in the global data, including the reactor anomaly, the tension in the $\Delta m_{21}^{2}$ determination from solar experiments versus KamLAND, the determination of $\Delta m_{31}^{2}$, tendencies in fit results for $\theta_{23}$ and $\delta_{\mathrm{CP}}$, and statistical issues related to the determination of the $\mathrm{CP}$ violating phase $\delta_{\mathrm{CP}}$. Finally in section 5 we present our conclusions.

The numerical results of our analysis as well as figures are available at the website [12], where also one- and two-dimensional $\chi^{2}$ tables are available for download. Furthermore, this website will be kept up-to-date when new data becomes available.

\section{Oscillation parameters: results of the global analysis}

\subsection{Data included in our analysis}

We include in our global analysis the results from Super-Kamiokande atmospheric neutrino data from phases SK1-4 [13], adding the 1775 days of phase SK4 to their published results on phases SK1-3 [14]. Concretely, we consider sub-GeV and multi-GeV $e$-like and $\mu$-like fully contained events, as well as partially contained, stopping and through-going $\mu$ data, each divided into 10 angular bins. Hence we have a total of 70 energy and zenith angle bins. For what concerns disappearance results from long baseline accelerator experiments (LBL) we use the energy distribution of events from MINOS in both $\nu_{\mu}\left(\bar{\nu}_{\mu}\right)$ disappearance with $10.71(3.36) \times 10^{20}$ protons on target (pot) [15], which amounts to 39 (14) data points, and from $\mathrm{T} 2 \mathrm{~K}$ in $\nu_{\mu}$ disappearance [16] with $6.57 \times 10^{20}$ pot (16 data points). For LBL appearance results we include both the neutrino and antineutrino events from 
MINOS [17], with exposure $10.6 \times 10^{20}$ and $3.3 \times 10^{20}$ pot, respectively, and from T2K in $\nu_{e}$ appearance [18] with $6.57 \times 10^{20}$ pot; each of these samples contributes 5 data points.

In the analysis of solar neutrino experiments we include the total rates from the radiochemical experiments Chlorine [19], Gallex/GNO [20] and SAGE [21]. For real-time experiments we include the results from on electron scattering (ES) from the four phases in Super-Kamiokande: the 44 data points of the phase I (SK1) energy-zenith spectrum [22], the 33 (42) data points of the full energy and day/night spectrum in phase II (III), SK2 [23] (SK3 [24]), and the 24 data points of the energy spectrum and day-night asymmetry of the 1669-day of phase IV, SK4 [25]. The results of the three phases of SNO are included in terms of the parametrization given in their combined analysis [26] which amount to 7 data points. We also include the main set of the 740.7 days of Borexino data [27] as well as their high-energy spectrum from 246 live days [28]. In the analysis of solar neutrino data we use the GS98 version of the solar standard model [29] (see section 4.2).

For oscillation signals at reactor experiments we include data from the finalized experiments CHOOZ [30] (energy spectrum data, 14 data points) and Palo Verde [31] (total rate) together with the spectrum from Double Chooz with 227.9 days live time [32] (18 data points), and the 621-day spectrum from Daya Bay [33] (36 data points), as well as the near and far rates observed at RENO with 800 days of data-taking [34] (2 data points with free normalization). We also include the observed energy spectrum in KamLAND data sets DS-1 and DS-2 [35] with a total exposure of $3.49 \times 10^{32}$ target-proton-year (2135 days). Although reactor experiments with baselines $\lesssim 100 \mathrm{~m}$ do not contribute to oscillation physics, they play an important role in constraining the unoscillated reactor neutrino flux. For this purpose we consider also data from Bugey4 [36], ROVNO4 [37], Bugey3 [38], Krasnoyarsk [39, 40], ILL [41], Gösgen [42], SRP [43], and ROVNO88 [44], to which we refer as reactor short-baseline experiments (RSBL). Details on the RSBL analysis can be found in [4].

For convenience a detailed list of all the data used in our global analysis can also be found in appendix A.

\subsection{Description of the results}

The results of the global analysis are presented in figures 1 and 2 where we show different projections of the allowed six-dimensional parameter space. To account for the possible effect of the so-called reactor anomaly [45-47], we follow the approach of refs. [48, 49] and study the dependence of the determined value of the parameters on the assumptions about the reactor fluxes. To bracket the possible impact of the anomaly, the results in figures 1 and 2 are shown for two extreme choices. The first option is to leave the normalization of reactor fluxes free and include data from short-baseline (less than $100 \mathrm{~m}$ ) reactor experiments. This corresponds to the colored regions in figure 1 and the solid curves in figure 2 (labeled "Free+RSBL"). The second option is not to include short-baseline reactor data but assume reactor fluxes as predicted in [45] (including their uncertainties). This corresponds to the black contours in figure 1 and the dashed curves in figure 2 (labeled "Huber"). From the results in these figures we conclude that: 


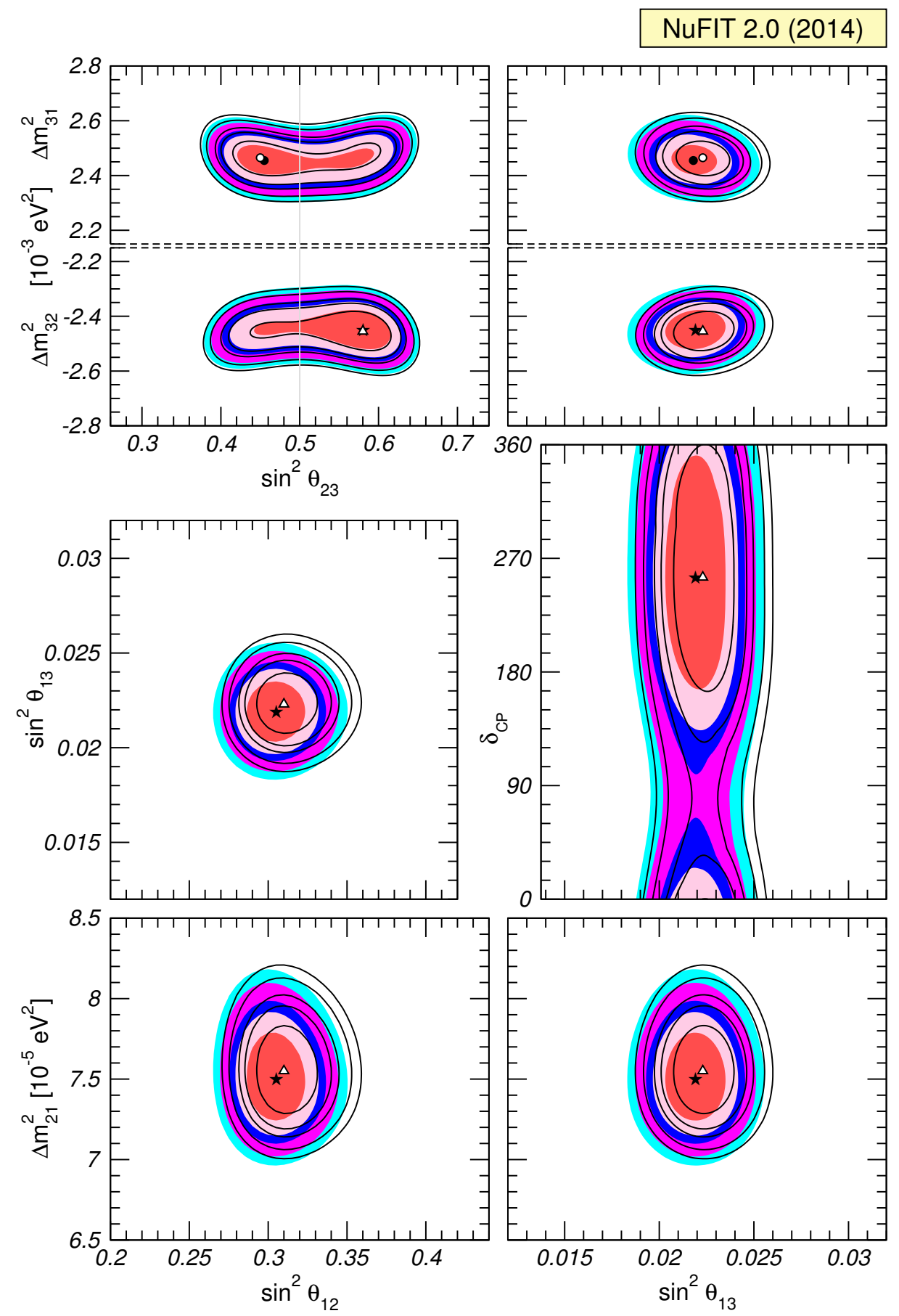

Figure 1. Global $3 \nu$ oscillation analysis. Each panel shows a two-dimensional projection of the allowed six-dimensional region after minimization with respect to the undisplayed parameters. The different contours correspond to $1 \sigma, 90 \%, 2 \sigma, 99 \%$ and $3 \sigma$ CL ( 2 dof). Full regions correspond to the analysis with free normalization of reactor fluxes and data from short-baseline (less than 100 $\mathrm{m}$ ) reactor experiments included. For void regions short-baseline reactor data are not included but reactor fluxes as predicted in [45] are assumed. Note that as atmospheric mass-squared splitting we use $\Delta m_{31}^{2}$ for $\mathrm{NO}$ and $\Delta m_{32}^{2}$ for IO. The regions in the lower 4 panels are based on a $\Delta \chi^{2}$ minimized with respect to $\mathrm{NO}$ and $\mathrm{IO}$. 

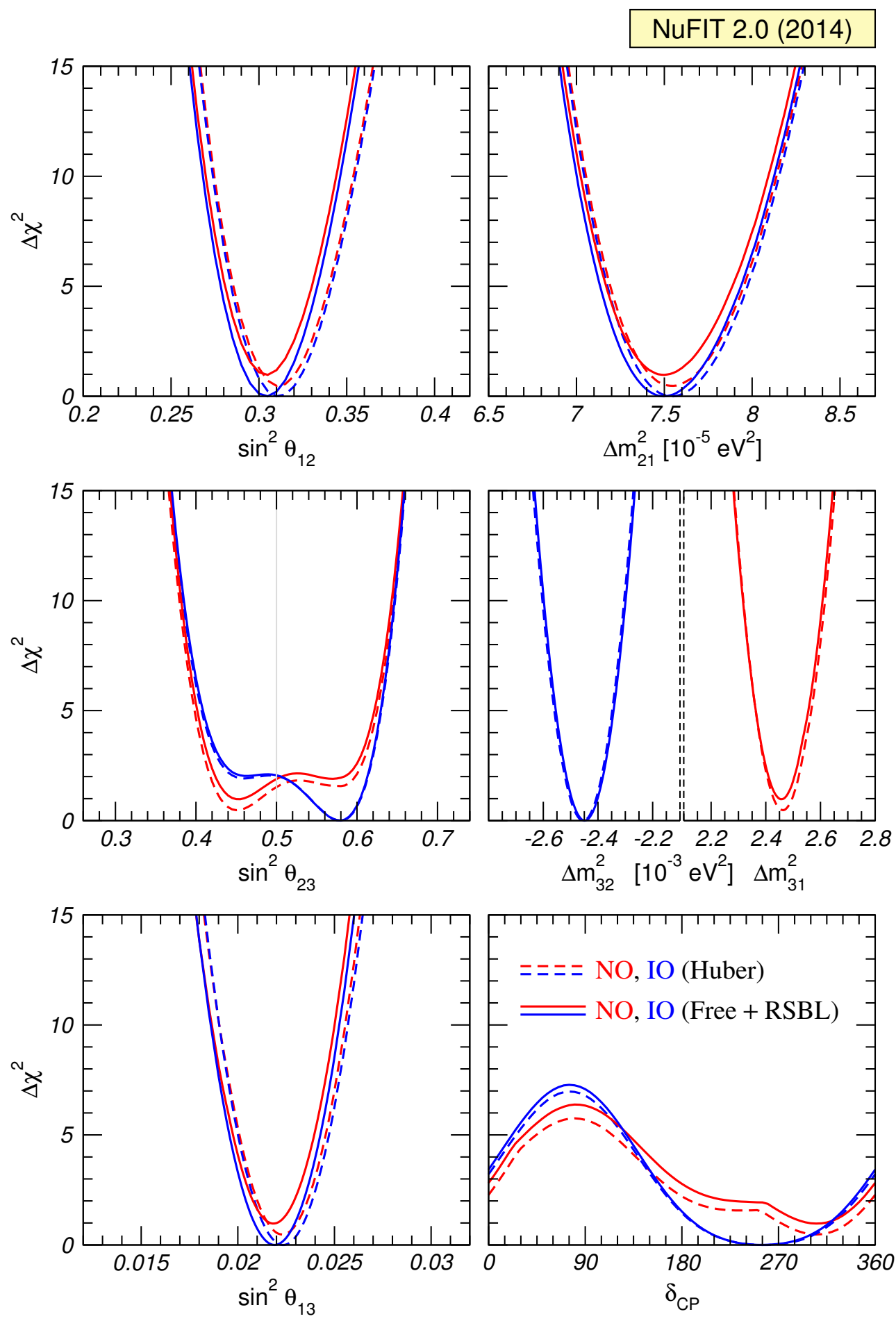

Figure 2. Global $3 \nu$ oscillation analysis. The red (blue) curves are for Normal (Inverted) Ordering. For solid curves the normalization of reactor fluxes is left free and data from short-baseline (less than $100 \mathrm{~m}$ ) reactor experiments are included. For dashed curves short-baseline data are not included but reactor fluxes as predicted in [45] are assumed. Note that as atmospheric mass-squared splitting we use $\Delta m_{31}^{2}$ for NO and $\Delta m_{32}^{2}$ for IO. 
1. for either choice of the reactor fluxes the global best fit corresponds to IO with $\sin ^{2} \theta_{23}>0.5$, while the second local minima is for $\mathrm{NO}$ and with $\sin ^{2} \theta_{23}<0.5$;

2. the statistical significance of the preference for Inverted versus Normal ordering is quite small, $\Delta \chi^{2} \lesssim 1 \sigma$

3. the present global analysis disfavors $\theta_{13}=0$ with a $\Delta \chi^{2} \approx 500$. Such impressive result is mostly driven by the reactor data from Daya Bay with secondary contributions from RENO and Double Chooz;

4. the uncertainty on $\theta_{13}$ associated with the choice of reactor fluxes is reduced to the level of $0.5 \sigma$ in the global analysis. This is so because the most precise results from Daya Bay and RENO are reactor flux normalization independent, as further discussed in section 4.1;

5. a non-maximal value of the $\theta_{23}$ mixing is slightly favored, at the level of $\sim 1.4 \sigma$ for Inverted Ordering at of $\sim 1.0 \sigma$ for Normal Ordering;

6. the statistical significance of the preference of the fit for the second (first) octant of $\theta_{23}$ is $\leq 1.4 \sigma(\leq 1.0 \sigma)$ for $\mathrm{IO}(\mathrm{NO})$;

7. the best fit for $\delta_{\mathrm{CP}}$ for all analyses and orderings occurs for $\delta_{\mathrm{CP}} \simeq 3 \pi / 2$, and values around $\pi / 2$ are disfavored with $\Delta \chi^{2} \simeq 6$. A discussion on the corresponding CL can be found in section 4.5 .

In what follows we will consider our default analysis choice the one with "Free Fluxes + RSBL". It is for this choice of fluxes that the best fit values and the derived ranges for the six parameters at the $1 \sigma(3 \sigma)$ level are given in table 1 . For each parameter the ranges are obtained after marginalizing with respect to the other parameters. We show the results for three scenarios. In the first and second columns we assume that the ordering of the neutrino mass states is known "a priori" to be Normal or Inverted, respectively, so the ranges of all parameters are defined with respect to the minimum in the given scenario. In the third column we make no assumptions on the ordering, so in this case the ranges of the parameters are defined with respect to the global minimum (which corresponds to Inverted Ordering) and are obtained marginalizing also over the ordering. For this third case we only give the $3 \sigma$ ranges. Of course in this case the range of $\Delta m_{3 \ell}^{2}$ is composed of two disconnected intervals, one one containing the absolute minimum (IO) and the other the secondary local minimum (NO).

Let us define the $3 \sigma$ relative precision of a parameter by $2\left(x^{\text {up }}-x^{\text {low }}\right) /\left(x^{\text {up }}+x^{\text {low }}\right)$, where $x^{\text {up }}\left(x^{\text {low }}\right)$ is the upper (lower) bound on a parameter $x$ at the $3 \sigma$ level. From the numbers in the table we then find $3 \sigma$ relative precisions of $14 \%\left(\theta_{12}\right), 32 \%\left(\theta_{23}\right), 15 \%\left(\theta_{13}\right)$, $14 \%\left(\Delta m_{21}^{2}\right)$ and $11 \%\left(\left|\Delta m_{3 \ell}^{2}\right|\right)$ for the various oscillation parameters.

\section{Mixing matrix and leptonic $\mathrm{CP}$ violation}

From the global $\chi^{2}$ analysis described in the previous section and following the procedure outlined in ref. [50] one can derive the $3 \sigma$ ranges on the magnitude of the elements of the 


\begin{tabular}{|l|cc|cc|c|}
\hline & \multicolumn{2}{|c|}{ Normal Ordering $\left(\Delta \chi^{2}=0.97\right)$} & \multicolumn{2}{|c|}{ Inverted Ordering (best fit) } & Any Ordering \\
\hline & bfp $\pm 1 \sigma$ & $3 \sigma$ range & bfp $\pm 1 \sigma$ & $3 \sigma$ range & $3 \sigma$ range \\
\hline $\sin ^{2} \theta_{12}$ & $0.304_{-0.012}^{+0.013}$ & $0.270 \rightarrow 0.344$ & $0.304_{-0.012}^{+0.013}$ & $0.270 \rightarrow 0.344$ & $0.270 \rightarrow 0.344$ \\
$\theta_{12} /^{\circ}$ & $33.48_{-0.75}^{+0.78}$ & $31.29 \rightarrow 35.91$ & $33.48_{-0.75}^{+0.78}$ & $31.29 \rightarrow 35.91$ & $31.29 \rightarrow 35.91$ \\
$\sin ^{2} \theta_{23}$ & $0.452_{-0.028}^{+0.052}$ & $0.382 \rightarrow 0.643$ & $0.579_{-0.037}^{+0.025}$ & $0.389 \rightarrow 0.644$ & $0.385 \rightarrow 0.644$ \\
$\theta_{23} /^{\circ}$ & $42.3_{-1.6}^{+3.0}$ & $38.2 \rightarrow 53.3$ & $49.5_{-2.2}^{+1.5}$ & $38.6 \rightarrow 53.3$ & $38.3 \rightarrow 53.3$ \\
$\sin ^{2} \theta_{13}$ & $0.0218_{-0.0010}^{+0.0010}$ & $0.0186 \rightarrow 0.0250$ & $0.0219_{-0.0010}^{+0.0011}$ & $0.0188 \rightarrow 0.0251$ & $0.0188 \rightarrow 0.0251$ \\
$\theta_{13} /^{\circ}$ & $8.50_{-0.21}^{+0.20}$ & $7.85 \rightarrow 9.10$ & $8.51_{-0.21}^{+0.20}$ & $7.87 \rightarrow 9.11$ & $7.87 \rightarrow 9.11$ \\
$\delta_{\mathrm{CP}}{ }^{\circ}$ & $306_{-70}^{+39}$ & $0 \rightarrow 360$ & $254_{-62}^{+63}$ & $0 \rightarrow 360$ & $0 \rightarrow 360$ \\
$\frac{\Delta m_{21}^{2}}{10^{-5} \mathrm{eV}^{2}}$ & $7.50_{-0.17}^{+0.19}$ & $7.02 \rightarrow 8.09$ & $7.50_{-0.17}^{+0.19}$ & $7.02 \rightarrow 8.09$ & $7.02 \rightarrow 8.09$ \\
$\frac{\Delta m_{3 \ell}^{2}}{10^{-3} \mathrm{eV}^{2}}$ & $+2.457_{-0.047}^{+0.047}$ & $+2.317 \rightarrow+2.607$ & $-2.449_{-0.047}^{+0.048}$ & $-2.590 \rightarrow-2.307$ & {$\left[\begin{array}{c}+2.325 \rightarrow+2.599 \\
-2.590 \rightarrow-2.307\end{array}\right]$} \\
\hline
\end{tabular}

Table 1. Three-flavor oscillation parameters from our fit to global data after the NOW 2014 conference. The results are presented for the "Free Fluxes + RSBL" in which reactor fluxes have been left free in the fit and short baseline reactor data (RSBL) with $L \lesssim 100 \mathrm{~m}$ are included. The numbers in the 1st (2nd) column are obtained assuming NO (IO), i.e., relative to the respective local minimum, whereas in the 3rd column we minimize also with respect to the ordering. Note that $\Delta m_{3 \ell}^{2} \equiv \Delta m_{31}^{2}>0$ for NO and $\Delta m_{3 \ell}^{2} \equiv \Delta m_{32}^{2}<0$ for IO.

leptonic mixing matrix to be:

$$
|U|=\left(\begin{array}{lll}
0.801 \rightarrow 0.845 & 0.514 \rightarrow 0.580 & 0.137 \rightarrow 0.158 \\
0.225 \rightarrow 0.517 & 0.441 \rightarrow 0.699 & 0.614 \rightarrow 0.793 \\
0.246 \rightarrow 0.529 & 0.464 \rightarrow 0.713 & 0.590 \rightarrow 0.776
\end{array}\right)
$$

By construction the derived limits in eq. (3.1) are obtained under the assumption of the matrix $U$ being unitary. In other words, the ranges in the different entries of the matrix are correlated due to the constraints imposed by unitarity, as well as the fact that, in general, the result of a given experiment restricts a combination of several entries of the matrix. As a consequence choosing a specific value for one element further restricts the range of the others.

The present status of the determination of leptonic CP violation is illustrated in figure 3 where we show the dependence of the $\Delta \chi^{2}$ of the global analysis on the Jarlskog invariant which gives a convention-independent measure of CP violation [51], defined as usual by:

$$
\operatorname{Im}\left[U_{\alpha i} U_{\alpha j}^{*} U_{\beta i}^{*} U_{\beta j}\right] \equiv \sum_{\gamma=e, \mu, \tau} \sum_{k=1,2,3} J_{\mathrm{CP}} \epsilon_{\alpha \beta \gamma} \epsilon_{i j k} \equiv J_{\mathrm{CP}}^{\max } \sin \delta_{\mathrm{CP}}
$$

Using the parametrization in eq. (1.1) we get

$$
J_{\mathrm{CP}}^{\max }=\cos \theta_{12} \sin \theta_{12} \cos \theta_{23} \sin \theta_{23} \cos ^{2} \theta_{13} \sin \theta_{13} .
$$




\section{NuFIT 2.0 (2014)}
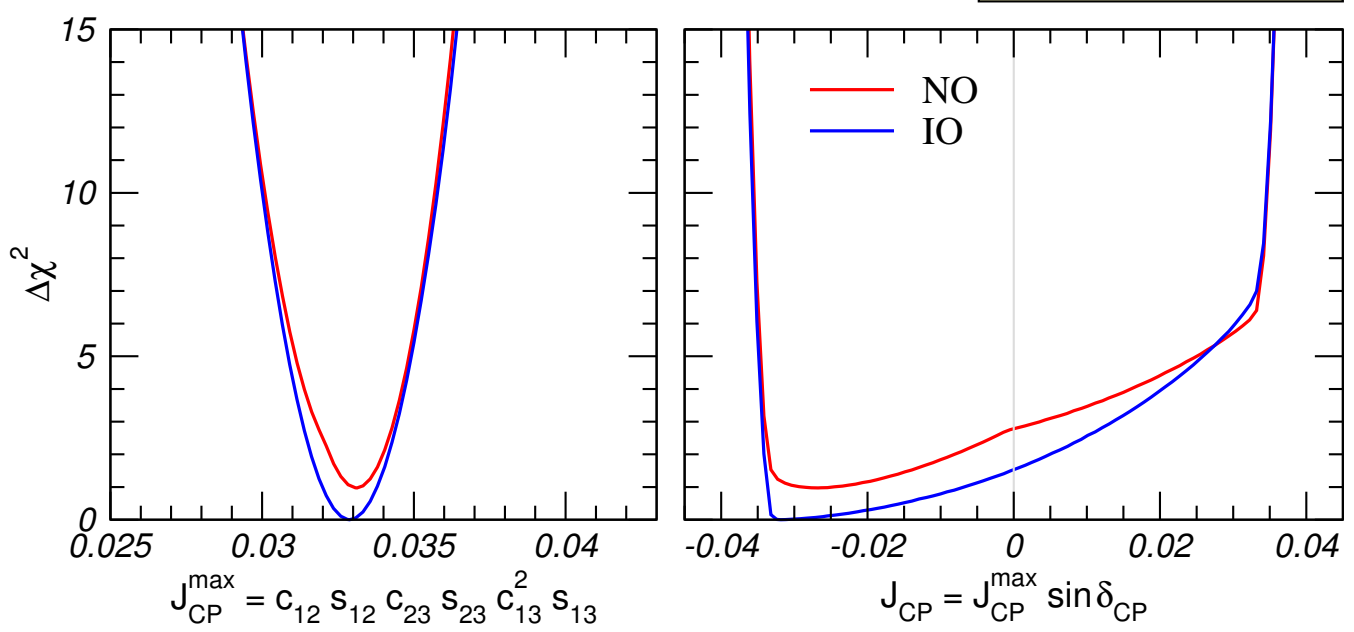

Figure 3. Dependence of the global $\Delta \chi^{2}$ function on the Jarlskog invariant. The red (blue) curves are for $\mathrm{NO}(\mathrm{IO})$.

From the left panel of figure 3 we see that the determination of the mixing angles yields at present a maximum allowed $\mathrm{CP}$ violation

$$
J_{\mathrm{CP}}^{\max }=0.033 \pm 0.010( \pm 0.027)
$$

at $1 \sigma(3 \sigma)$ for both orderings. The preference of the present data for non-zero $\delta_{\mathrm{CP}}$ implies a best fit $J_{\mathrm{CP}}^{\text {best }}=-0.033$, which is favored over CP conservation at the $\sim 1.3 \sigma$ level. These numbers can be compared with the size of the Jarlskog invariant in the quark sector, which is determined to be $J_{\text {CP }}^{\text {quarks }}=\left(2.96_{-0.16}^{+0.20}\right) \times 10^{-5}[7]$.

In figure 4 we recast the allowed regions for the leptonic mixing matrix in terms of leptonic unitarity triangles, which are obtained as different combinations of the entries of the $U$ matrix. ${ }^{1}$ Since in our analysis $U$ is unitary by construction, any given pair of rows or columns can be used to define a triangle in the complex plane. On the left (right) panels we show the triangles corresponding to the unitarity conditions

$$
\begin{array}{lll}
\sum_{i=1,2,3} U_{\alpha i} U_{\beta i}^{*}=0 & \text { with } \alpha \neq \beta & \text { (left), } \\
\sum_{\alpha=e, \mu, \tau} U_{\alpha i} U_{\alpha j}^{*}=0 & \text { with } i \neq j & \text { (right). }
\end{array}
$$

In drawing these triangles we have rescaled and rotated their sides so that two of their vertices always coincide with $(0,0)$ and $(1,0)$ in the complex plane. To this aim we have defined a complex variable $z$ as follows:

$$
\begin{aligned}
& z=-\frac{U_{\alpha i} U_{\beta i}^{*}}{U_{\alpha k} U_{\beta k}^{*}}=1+\frac{U_{\alpha j} U_{\beta j}^{*}}{U_{\alpha k} U_{\beta k}^{*}} \quad \text { with } \alpha \neq \beta \text { and } i \neq j \neq k \quad \text { (left), } \\
& z=-\frac{U_{\alpha i} U_{\alpha j}^{*}}{U_{\gamma i} U_{\gamma j}^{*}}=1+\frac{U_{\beta i} U_{\beta j}^{*}}{U_{\gamma i} U_{\gamma j}^{*}} \quad \text { with } i \neq j \text { and } \alpha \neq \beta \neq \gamma \quad \text { (right) }
\end{aligned}
$$

\footnotetext{
${ }^{1}$ See, e.g., refs. [52-55] for discussions of leptonic unitarity triangles.
} 

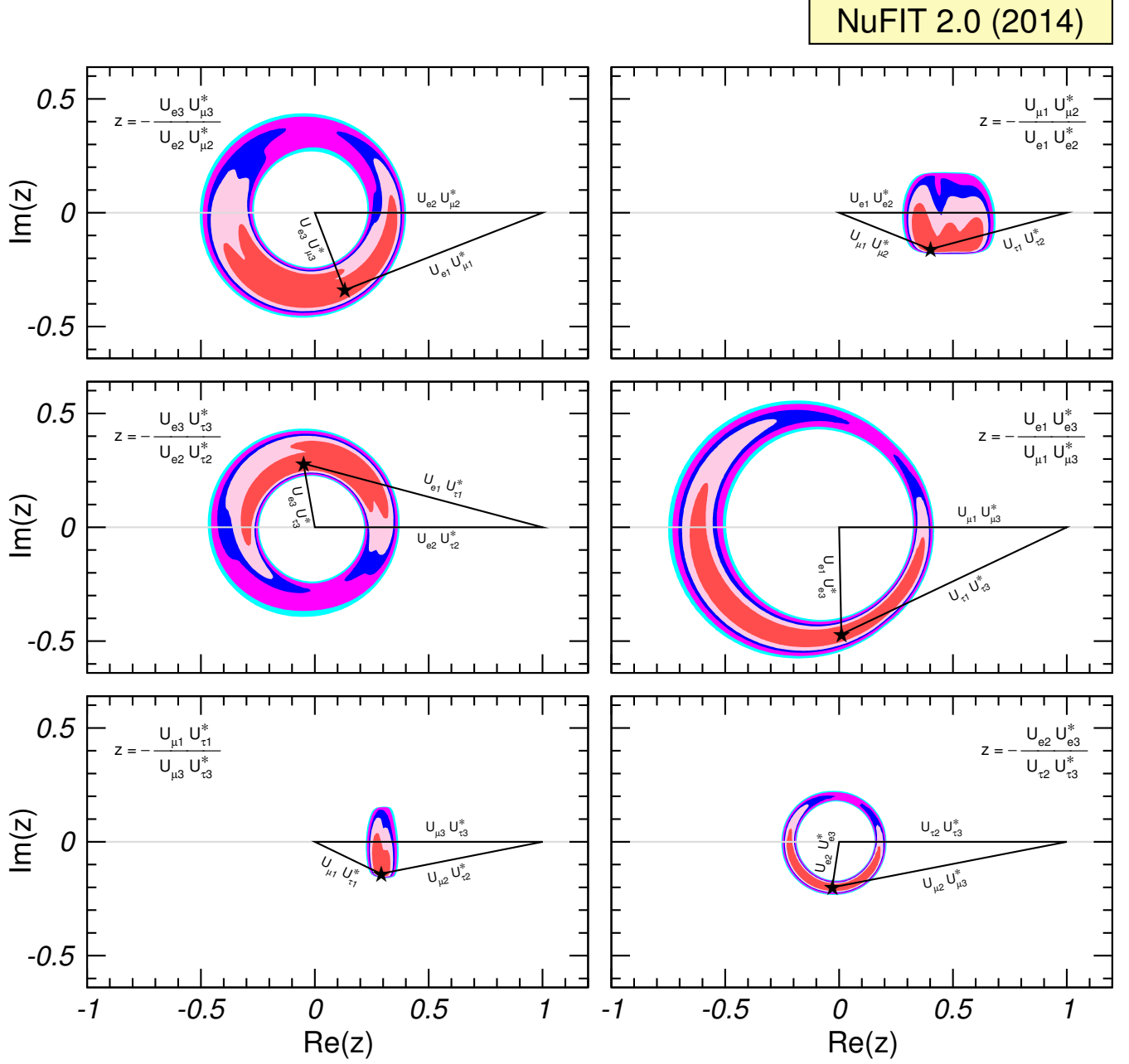

Figure 4. Six leptonic unitarity triangles. After scaling and rotating each triangle so that two of its vertices always coincide with $(0,0)$ and $(1,0)$ (see text for details) we plot the $1 \sigma, 90 \%, 2 \sigma, 99 \%$, $3 \sigma$ CL (2 dof) allowed regions of the third vertex. Note that in the construction of the triangles the unitarity of the $U$ matrix is always explicitly imposed.

and then we have plot the $1 \sigma, 90 \%, 2 \sigma, 99 \%, 3 \sigma$ CL ( 2 dof) allowed regions of the third vertex of the triangle as the real and imaginary parts of $z$. For convenience in each panel we have chosen the normalization side (the one which lies on the horizontal $(0,0) \rightarrow(0,1)$ segment) as the best determined of the two longer sides of each triangle. In this way all the triangles have more or less the same size, and the uncertainty in the position of the third vertex is not too much affected by the uncertainty of the normalization side. Note that the most common unitarity triangle in the quark sector is the one based on the $d$-quark and $b$-quark columns [7], which corresponds to the 1st and 3rd column in the leptonic matrix, i.e., to the triangle in the middle-right panel in figure 4.

In this kind of diagrams the absence of $\mathrm{CP}$ violation implies a flat triangle, i.e., $\operatorname{Im}(z)=$ 0 . As can be seen, in all the panels the horizontal axis marginally crosses the $1 \sigma$ allowed region, which for 2 dof corresponds to $\Delta \chi^{2} \simeq 2.3$. This is consistent with the present preference for CP violation, $\chi^{2}\left(J_{\mathrm{CP}}=0\right)-\chi^{2}\left(J_{\mathrm{CP}}\right.$ free $)=1.7$. 


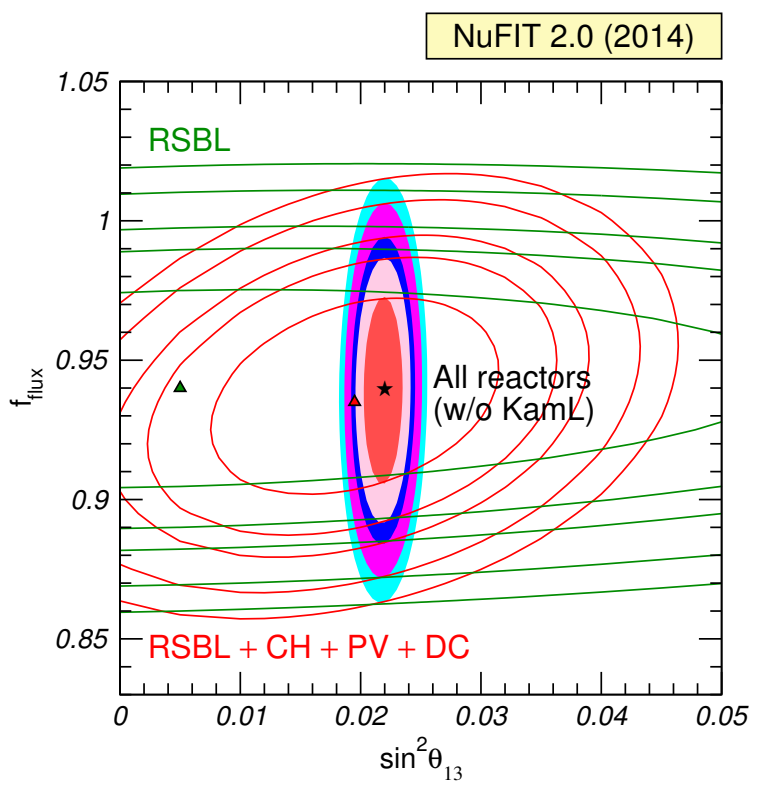

Figure 5. Contours $(1 \sigma, 90 \%, 2 \sigma, 99 \%, 3 \sigma$ CL for 2 dof $)$ in the plane of $\theta_{13}$ and the reactor flux normalization $f_{\text {flux }}$. Full regions correspond to the combined analysis of all reactor neutrino experiments with the exception of KamLAND, but including the RSBL experiments. The green contours correspond to only the RSBL experiments and red contours include RSBL + mediumbaseline reactors without a near detector (i.e. without including Daya Bay and RENO).

\section{Tension and tendencies}

\subsection{Impact of reactor flux uncertainties}

Within the 3-flavor framework the so-called reactor anomaly leads to a "tension" of about $2.7 \sigma$ between the predicted reactor neutrino fluxes $[45,46]$ and the event rates observed in short-baseline reactor experiments. By adopting two extreme approaches in dealing with this tension we have shown in section 2.2 that the impact on the determination of the oscillation parameters in the global fit is quite small, at the level of $0.5 \sigma$ for $\sin ^{2} \theta_{13}$ (see figures 1 and 2). This is further illustrated in figure 5 where we show the allowed regions in the plane of $\theta_{13}$ and the flux normalization $f_{\text {flux }}$ (relative to the one predicted in [45]) for several combinations of the reactor experiments. Short-baseline data (green contours) essentially determine the flux normalization. Adding also data from experiments at around $1 \mathrm{~km}$ without a dedicated near detector (red contours) provides already a signal for non-zero $\theta_{13}$, but such result is affected by significant correlation with the flux normalization. However, once the precise data on near-far comparison from Daya Bay and RENO are included (colored regions) no correlation is left between the determination of $\theta_{13}$ and $f_{\text {flux }}$. Thus in the $3 \nu$ analysis the unexplained reactor anomaly mostly translates in an overall increase of the $\chi^{2}$ in the analysis with fluxes from ref. [45] with $\chi^{2}\left(f_{\text {flux }}=1\right)-\chi^{2}\left(f_{\text {flux }}\right.$ free $) \simeq 7$. Details of our analysis in this respect can be found in ref. [4], where a discussion of a possible explanation in terms of sterile neutrinos is also given. 

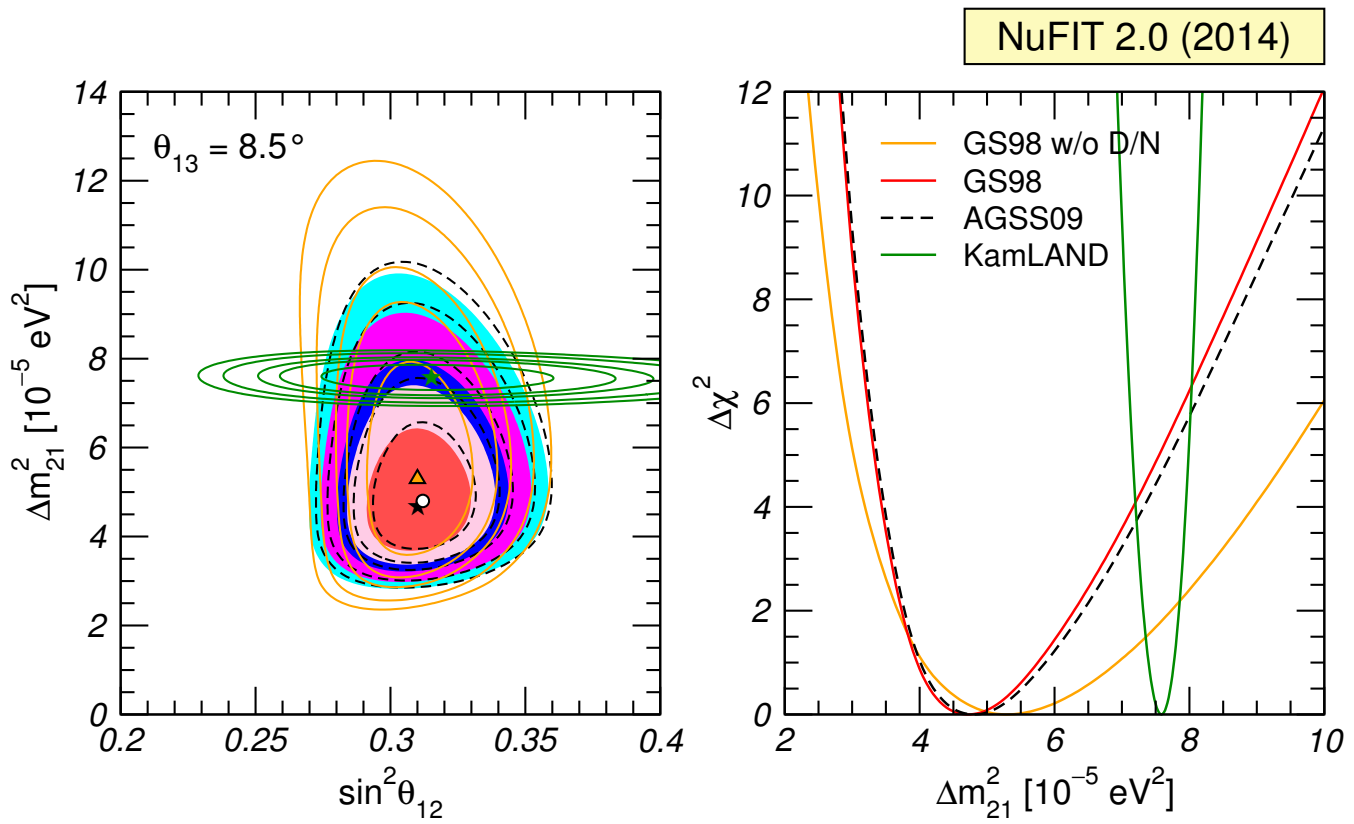

Figure 6. Left: allowed parameter regions (at $1 \sigma, 90 \%, 2 \sigma, 99 \%$ and $3 \sigma$ CL for 2 dof) from the combined analysis of solar data for GS98 model (full regions with best fit marked by black star) and AGSS09 model (dashed void contours with best fit marked by a white dot), and for the analysis of KamLAND data (solid green contours with best fit marked by a green star) for fixed $\theta_{13}=8.5^{\circ}$. We also show as orange contours the results of a global analysis for the GS98 model but without including the day-night information from SK (see text for details). Right: $\Delta \chi^{2}$ dependence on $\Delta m_{21}^{2}$ for the same four analysis after marginalizing over $\theta_{12}$.

\subsection{Determination of $\Delta m_{21}^{2}:$ solar and KamLAND}

We show in figure 6 the results of the analysis of the solar experiments and of KamLAND which give the dominant contribution to the determination of $\Delta m_{21}^{2}$ and $\theta_{12}$. Here $\theta_{13}$ is fixed to the present best fit value of the global analysis. For the sake of completeness the solar neutrino results are shown for two different versions of the Standard Solar Model, namely the GS98 and the AGSS09 models [29]. Let us remind that GS98 is based on the older solar abundances leading to high metallicity and which perfectly agreed with helioseismological data, whereas AGSS09 uses the new precise determination of the solar abundances which imply a lower metallicity and cannot reproduce the helioseismological data. This conflict constitutes the so-called "solar composition problem". Although it is a pretty serious problem in the context of solar physics, its impact in the determination of the relevant oscillation parameters is very small, as can be seen clearly from figure 6 .

The left panel in figure 6 illustrates the complementarity of solar and KamLAND in the determination of the " 12 " parameters. Solar experiments provide the best precision of $\theta_{12}$ while KamLAND gives a better determination of $\Delta m_{21}^{2}$. We remind the reader that the relevant survival probabilities for these experiments in the framework of three neutrino oscillations can be written as:

$$
P_{e e}^{3 \nu}=\sin ^{4} \theta_{13}+\cos ^{4} \theta_{13} P_{e e}^{2 \nu}\left(\Delta m_{21}^{2}, \theta_{12}\right),
$$


where we have used the fact that $L_{31}^{\text {osc }}=4 \pi E_{\nu} / \Delta m_{31}^{2}$ is much shorter than the distance traveled by both solar and KamLAND neutrinos, so that the oscillations related to $L_{31}^{\text {osc }}$ are averaged. In presence of matter effects $P_{e e}^{2 \nu}\left(\Delta m_{21}^{2}, \theta_{12}\right)$ should be calculated taking into account the evolution in an effective matter density $n_{e}^{\text {eff }}=n_{e} \cos ^{2} \theta_{13}$. For $10^{-5} \lesssim$ $\Delta m^{2} / \mathrm{eV}^{2} \lesssim 10^{-4}, P_{e e}^{2 \nu}\left(\Delta m_{21}^{2}, \theta_{12}\right)$ presents the following asymptotic behaviors [56]:

$$
\begin{array}{ll}
P_{e e}^{2 \nu, \text { sun }} \simeq 1-\frac{1}{2} \sin ^{2}\left(2 \theta_{12}\right) & \text { for } E_{\nu} \lesssim \text { few } \times 100 \mathrm{KeV} \\
P_{e e}^{2 \nu, \text { sun }} \simeq \sin ^{2}\left(\theta_{12}\right) & \text { for } E_{\nu} \gtrsim \text { few } \times 1 \mathrm{MeV} \\
P_{e e}^{2 \nu, \text { kam }}=1-\frac{1}{2} \sin ^{2}\left(2 \theta_{12}\right) \sin ^{2} \frac{\Delta m_{21}^{2} L}{2 E_{\nu}} . &
\end{array}
$$

At present most of the precision of the solar analysis is provided by SNO and SK for which the relevant MSW survival probability $[57,58]$ provides a direct measurement of $\sin ^{2} \theta_{12}$, as seen in eq. (4.3). In the MSW regime the determination of $\Delta m_{21}^{2}$ in solar experiments comes dominantly from the ratio between the solar potential and the $\Delta m_{21}^{2}$ term required to simultaneously describe the $\mathrm{CC} / \mathrm{NC}$ data at $\mathrm{SNO}$ and the undistorted spectra of ${ }^{8} \mathrm{~B}$ neutrinos as measured in both SK and SNO. Conversely KamLAND $\bar{\nu}_{e}$ survival probability proceeds dominantly as vacuum oscillations and provides a most precise determination of $\Delta m_{21}^{2}$ via the strong effect of the oscillating phase in the distortion of the reactor energy spectrum. On the contrary it yields a weaker constraint on $\theta_{12}$ as the vacuum oscillation probability depends on the double-valued and "flatter" function $\sin ^{2}\left(2 \theta_{12}\right)$.

As seen in the left panel in figure 6 for either version of the solar model the best fit points of solar and KamLAND analysis lie at very similar values of $\theta_{12}$. As it was pointed out in ref. [59] and widely discussed in the literature [60-64], the matching in the determination of $\theta_{12}$ requires the presence of a non-zero value of $\theta_{13}$. With the present determination of $\theta_{13}$ provided by the medium baseline reactor experiments, the agreement between the best fit point values of $\theta_{12}$ is remarkable.

From the same figure, however, we see that the value of $\Delta m_{21}^{2}$ preferred by KamLAND is higher than the one from solar experiments. At present this is about a $2 \sigma$ effect, as can be seen in the right panel where we show the $\Delta \chi^{2}$ dependence as a function of $\Delta m_{21}^{2}$ when marginalized over $\theta_{12}$. This tension has been present during the last two years and it arises from a combination of two effects: (a) the well-known fact that none of the ${ }^{8} \mathrm{~B}$ measurement performed by SNO, SK and Borexino show any evidence of the spectrum low energy turnup expected in the standard LMA-MSW solution, and (b) the indication of a non-vanishing day-night asymmetry in SK, which disfavors the KamLAND $\Delta m_{21}^{2}$ best fit value for which Earth matter effects are too small. The relevance of these effects is illustrated in figure 6 where we show the results of our analysis both with and without the inclusion of the SK day-night information. As can be seen, once the SK day-night information is removed the solar best-fit point shifts upwards and the solar allowed region extends to much larger values of $\Delta m_{21}^{2}$, as expected, so that the tension with KamLAND is reduced to about $1.4 \sigma$. Modified matter potential due to non-standard interactions $[65,66]$ and super-light sterile neutrinos [67] have been proposed as extended scenarios which could relax this tension. 

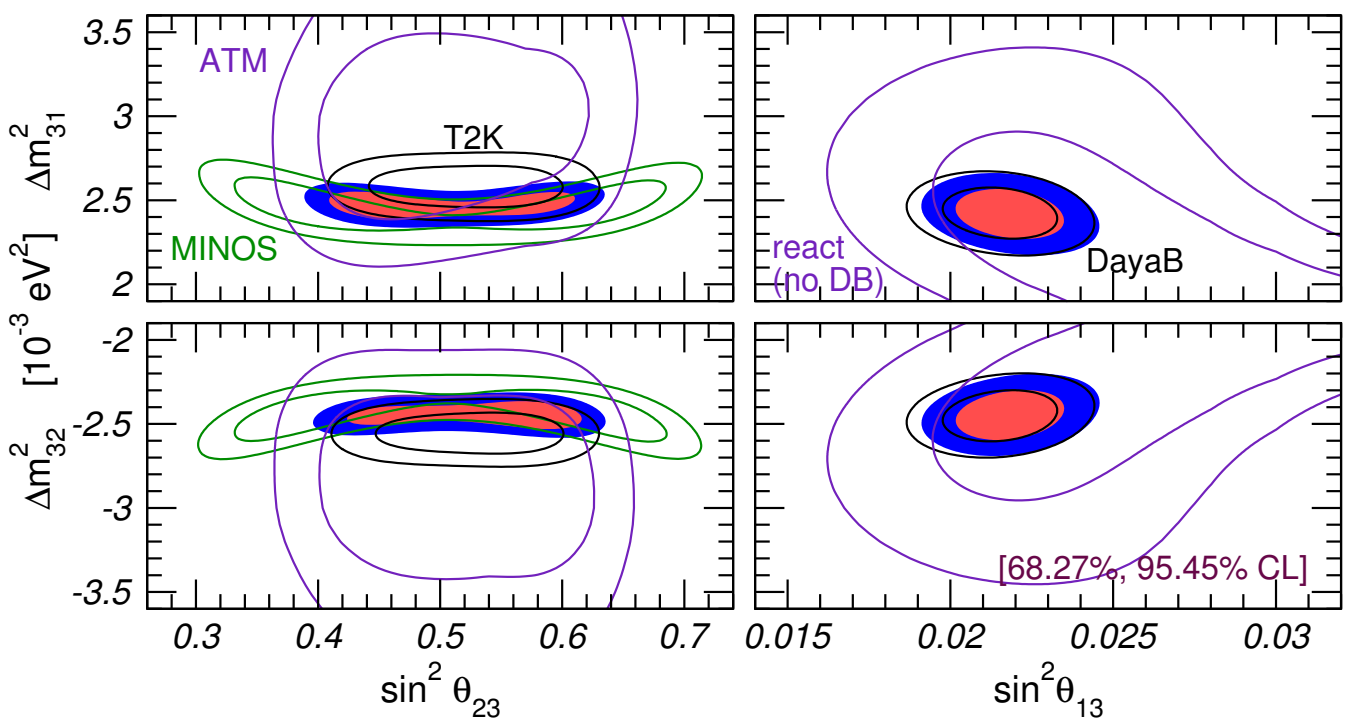

Figure 7. Determination of $\Delta m_{3 \ell}^{2}$ at $1 \sigma$ and $2 \sigma$ ( 2 dof), where $\ell=1$ for NO (upper panels) and $\ell=2$ for IO (lower panels). The left panels show regions in the $\left(\sin ^{2} \theta_{23}, \Delta m_{3 \ell}^{2}\right)$ plane using both appearance and disappearance data from MINOS (green) and T2K (black), as well as SK atmospheric data (green) and a combination of them (colored regions). Here $\theta_{13}$ is constrained to the $3 \sigma$ range from the global fit. The right panels show regions in the $\left(\sin ^{2} \theta_{13}, \Delta m_{3 \ell}^{2}\right)$ plane using data from Daya Bay (black), reactor data without Daya Bay (violet), and their combination (colored regions). In all panels solar and KamLAND data are included to constrain $\Delta m_{21}^{2}$ and $\theta_{12}$. Contours are defined with respect to the local minimum in each panel.

\subsection{Determination of $\Delta m_{3 \ell}^{2}: \nu_{\mu}$ and $\nu_{e}$ disappearance}

Figure 7 illustrates the determination of $\Delta m_{3 \ell}^{2}$ from different data sets. In the left panels we focus on long-baseline $\nu_{\mu}$ disappearance data. It is clear that in this case the final precision on $\left|\Delta m_{3 \ell}^{2}\right|$ emerges from the combination of T2K and MINOS data, while the determination of $\sin ^{2} \theta_{23}$ is dominated by $\mathrm{T} 2 \mathrm{~K}$.

Concerning $\nu_{e}$ disappearance data, eq. (4.8) in section 4.4 implies that the rates observed in reactor experiments at different baselines can provide an independent determination of $\Delta m_{3 \ell}^{2}[49,68]$. On top of this, the observation of the energy-dependent oscillation effect of $\theta_{13}$ in Daya Bay [69] allows a rather precise determination of $\left|\Delta m_{3 \ell}^{2}\right|$. In the right panels of figure 7 we show therefore the allowed regions in the $\left(\theta_{13}, \Delta m_{3 \ell}^{2}\right)$ plane based on global data on $\nu_{e}$ disappearance. The blue contours are obtained from all the mediumbaselines reactor experiments with the exception of Daya Bay. Those regions emerge from the baseline effect mentioned above. The black contour are based on the energy spectrum in Daya Bay, whereas the colored regions show the combination.

By comparing the left and right panels we observe that $\nu_{\mu}$ and $\nu_{e}$ disappearance experiments by now provide a consistent determination of $\left|\Delta m_{3 \ell}^{2}\right|$ with similar precision. 


\subsection{Mass ordering, $\theta_{23}$ octant and CP phase: role of different data sets}

As we have seen in section 2, several 1-2 $\sigma$ "tendencies" appear in the global analysis in the determination of the mass ordering, the octant of $\theta_{23}$, and the CP violating phase. To illustrate the role of the different data sets on such tendencies, we show in figure 8 the $\Delta \chi^{2}$ as a function of $\Delta m_{3 \ell}^{2}, \theta_{23}$, and $\delta_{\mathrm{CP}}$ for different combinations of experiments. In each panel the results have been marginalized with respect to all undisplayed parameters except the mass ordering, which is fixed to Inverted (Normal) for the left (right) panels. Note, however, that for each combination of experiments the $\Delta \chi^{2}$ is defined with respect to the absolute minima between the two orderings. In this way the difference between the "height" of the minimum of the curve on the left and the corresponding one on the right gives the contribution of that set of observables to the determination of the mass ordering.

All the lines plotted in figure 8 include "by default" solar and reactor data, which take care of precisely determining the undisplayed parameters $\Delta m_{21}^{2}, \theta_{12}$ and $\theta_{13}$. To this basic set we progressively add more and more data, to see how each new piece of information affects the results of the fit. Let us then start with the dotted purple (and dot-dashed blue) curve, which shows the dependence of $\Delta \chi^{2}$ on the analysis of solar, reactor and MINOS (plus T2K) $\nu_{\mu}$ and $\bar{\nu}_{\mu}$ disappearance data. Being all disappearance experiments they provide very weak information on $\delta_{\mathrm{CP}}$, as clearly visible in the bottom panels. Comparing the minima in the left and right panels we note a relative difference of $\chi^{2}(\mathrm{NO})-\chi^{2}(\mathrm{IO}) \sim$ 0.2 , which means that this combination of data "favors" Inverted Ordering by $\sim 0.5 \sigma$. More interestingly, from the central panels we see that MINOS disappearance data favors a non-maximal $\theta_{23}$ with $\Delta \chi^{2}\left(\theta_{23}=45^{\circ}\right)=2.8(2.2)$ for IO (NO). Neglecting subleading $\Delta m_{21}^{2}$ and matter effects, the relevant survival probability in MINOS is given by

$$
P_{\nu_{\mu} \rightarrow \nu_{\mu}} \approx 1-\sin ^{2} 2 \theta_{\text {dis }} \sin ^{2} \frac{\Delta m_{31}^{2} L}{4 E_{\nu}}, \quad \sin ^{2} \theta_{\text {dis }} \equiv \sin ^{2} \theta_{23} \cos ^{2} \theta_{13},
$$

where $L$ is the baseline and $E_{\nu}$ is the neutrino energy. Hence, the probability is symmetric under $\theta_{\text {dis }} \rightarrow \pi / 2-\theta_{\text {dis }}$. In the limit $\theta_{13}=0$ the effective angle $\theta_{\text {dis }}$ reduces to $\theta_{23}$, and a preference for non-maximal $\theta_{\text {dis }}$ mixing leads to the appearance of two symmetric minima in the first and second octant of $\theta_{23}$. Such degeneracy persists also for $\theta_{13} \neq 0$, and is responsible for the presence of two quasi-degenerate minima at $\sin ^{2} \theta_{23}=0.63$ and 0.39 . On the other hand, T2K disappearance data are better fitted with maximal $\theta_{\text {dis }}$, so once they are included in the analysis (dot-dashed blue line) the positions of the two minima move to values $\sin ^{2} \theta_{23}=0.58$ and 0.44 while the preference for non-maximal mixing reduces to $\Delta \chi^{2}\left(\theta_{23}=45^{\circ}\right)=1$. The comparison of the dotted purple and dot-dashed blue curves also shows the impact of the inclusion of $\mathrm{T} 2 \mathrm{~K}$ disappearance data on the overall determination of $\theta_{23}$ and $\Delta m_{3 \ell}^{2}$.

The short-dashed green line shows the effect of further adding to the analysis the T2K $\nu_{e}$ appearance data. First, we see that the absolute minima now occurs for NO with $\Delta \chi^{2}(\mathrm{IO})=0.6$. In the central panels we see that the quasi-degeneracy of the octant of $\theta_{23}$ is now broken and the second octant becomes favored with $\Delta \chi^{2}\left(\theta_{23} \leq 45^{\circ}\right)=2.5(1.5)$ for IO (NO). The lower panels show that after the inclusion of $\mathrm{T} 2 \mathrm{~K} \nu_{e}$ appearance data a minimum appears for $\delta_{\mathrm{CP}}=270^{\circ}\left(300^{\circ}\right)$ for IO (NO) with CP conservation disfavored 

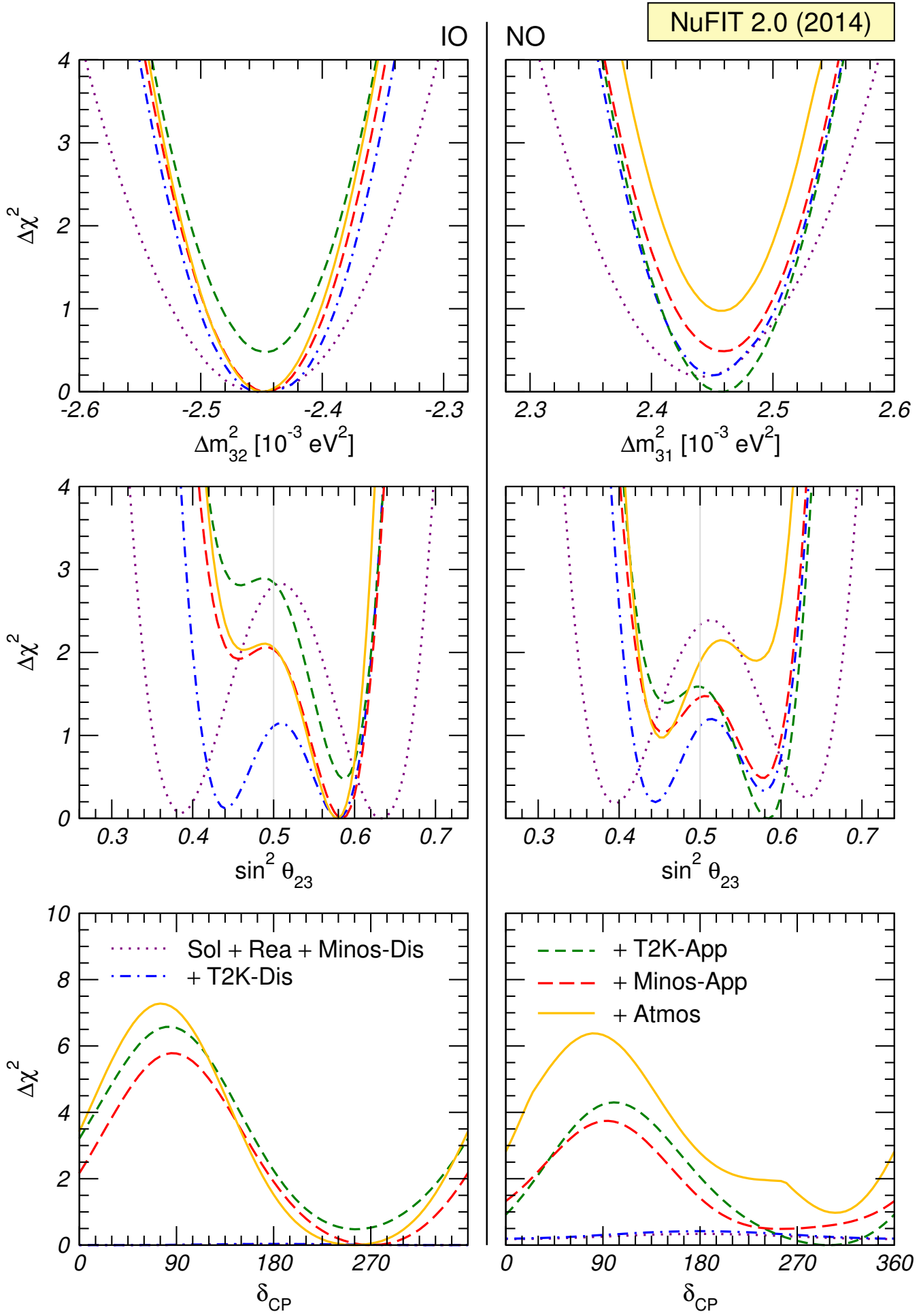

Figure 8. Contribution of different sets of experimental results to the present tendencies in the determination of the mass ordering, the octant of $\theta_{23}$ and of the $\mathrm{CP}$ violating phase. Left (right) panels are for IO (NO). See text for details. 
at $\Delta \chi^{2}\left(\sin \delta_{\mathrm{CP}}=0\right)=2.5(1.0)$. This can be understood from the relevant $\nu_{e}$ appearance probability at $\mathrm{T} 2 \mathrm{~K}$ and MINOS, which, at the second order in the small parameters $\sin \theta_{13}$ and $\alpha \equiv \Delta m_{21}^{2} / \Delta m_{31}^{2}$ and assuming a constant matter density, takes the form [70-72]:

$$
\begin{aligned}
P_{\nu_{\mu} \rightarrow \nu_{e}} \approx & 4 \sin ^{2} \theta_{13} \sin ^{2} \theta_{23} \frac{\sin ^{2} \Delta(1-A)}{(1-A)^{2}}+\alpha^{2} \sin ^{2} 2 \theta_{12} \cos ^{2} \theta_{23} \frac{\sin ^{2} A \Delta}{A^{2}} \\
& +2 \alpha \sin \theta_{13} \sin 2 \theta_{12} \sin 2 \theta_{23} \cos \left(\Delta \pm \delta_{\mathrm{CP}}\right) \frac{\sin \Delta A}{A} \frac{\sin \Delta(1-A)}{1-A},
\end{aligned}
$$

with

$$
\Delta \equiv \frac{\Delta m_{31}^{2} L}{4 E_{\nu}}, \quad A \equiv \frac{2 E_{\nu} V}{\Delta m_{31}^{2}} .
$$

Here $L$ is the baseline, $E_{\nu}$ is the neutrino energy, and $V$ is the effective matter potential [57] which for T2K yields $|A| \sim$ few $\%$. The first term in eq. (4.6) (which dominates for large $\left.\theta_{13}\right)$ depends on $\sin ^{2} \theta_{23}$ and therefore is sensitive to the octant. Reactor experiments with $L \sim 1 \mathrm{~km}$, on the other hand, provide a measurement of $\theta_{13}$ independent of $\theta_{23}$

$$
P_{\nu_{e} \rightarrow \nu_{e}}=1-\sin ^{2} 2 \theta_{13} \sin ^{2} \frac{\Delta m_{31}^{2} L}{4 E_{\nu}}+\mathcal{O}\left(\alpha^{2}\right)
$$

At present the $\nu_{e}$ appearance results from $\mathrm{T} 2 \mathrm{~K}$ points towards an excess with respect to what is expected for the best fit value of $\sin ^{2} \theta_{13}$ determined by the reactor experiments for maximal $\theta_{23}$ (i.e., for $2 \sin ^{2} \theta_{23}=1$ ), hence the tendency towards the $\theta_{23}>45^{\circ}$ minimum. The matter effects in eq. (4.6) make this tendency different for NO and IO, while the last term introduces a $\delta_{\mathrm{CP}}$ modulation of the effect. For fixed $\theta_{13}$ and $\theta_{23}$, $P_{\nu_{\mu} \rightarrow \nu_{e}}\left(\delta_{\mathrm{CP}}\right)-P_{\nu_{\mu} \rightarrow \nu_{e}}(\pi) \geq 0(\leq 0)$ for $\delta_{\mathrm{CP}} \geq \pi(\leq \pi)$. For the best fit values of $\theta_{13}$ and $\theta_{23}$ from the previous reactor and LBL $\nu_{\mu}$ disappearance results, the T2K $\nu_{e}$ appearance signal is better fitted with $\delta_{\mathrm{CP}}$ values which enhance the corresponding appearance probability. Conversely we see that adding the less significant MINOS $\nu_{e}$ appearance data in the analysis (long-dashed red curves) tends to slightly reduce the size of these effects for $\mathrm{NO}$ and it shifts the global minimum from NO to IO.

Finally the solid orange curves show the impact of including the atmospheric data in the analysis. Comparing the solid orange and long-dashed red curves we see that atmospheric data contributes positively to the significance of the tendency towards IO and $\delta_{\mathrm{CP}}>\pi$. While for IO it does not affect the tendency towards second $\theta_{23}$ octant, for NO it "shifts" this tendency to the first octant. The preference for $\theta_{23}<45^{\circ}$ for NO is related to an excess of sub-GeV e-like events, an effect which has already been discussed since many years (see, e.g., [73-76]). The fact that this preference is not visible for IO is probably related to multi$\mathrm{GeV}$ data, which are affected by matter effects and therefore provides some sensitivity to the mass ordering. Identifying the relevant bins is difficult, given the large amount of data points entering the atmospheric fit. We stress that such effects happen at the level of 1-2 units in $\chi^{2}$ and hence are not statistically significant. ${ }^{2}$

\footnotetext{
${ }^{2}$ In this respect it is also important to stress that already since SK2 the Super-Kamiokande collaboration has been presenting its experimental results in terms of a large number of data samples. The rates for some of those samples cannot be theoretically predicted (and therefore included in a statistical analysis) without
} 

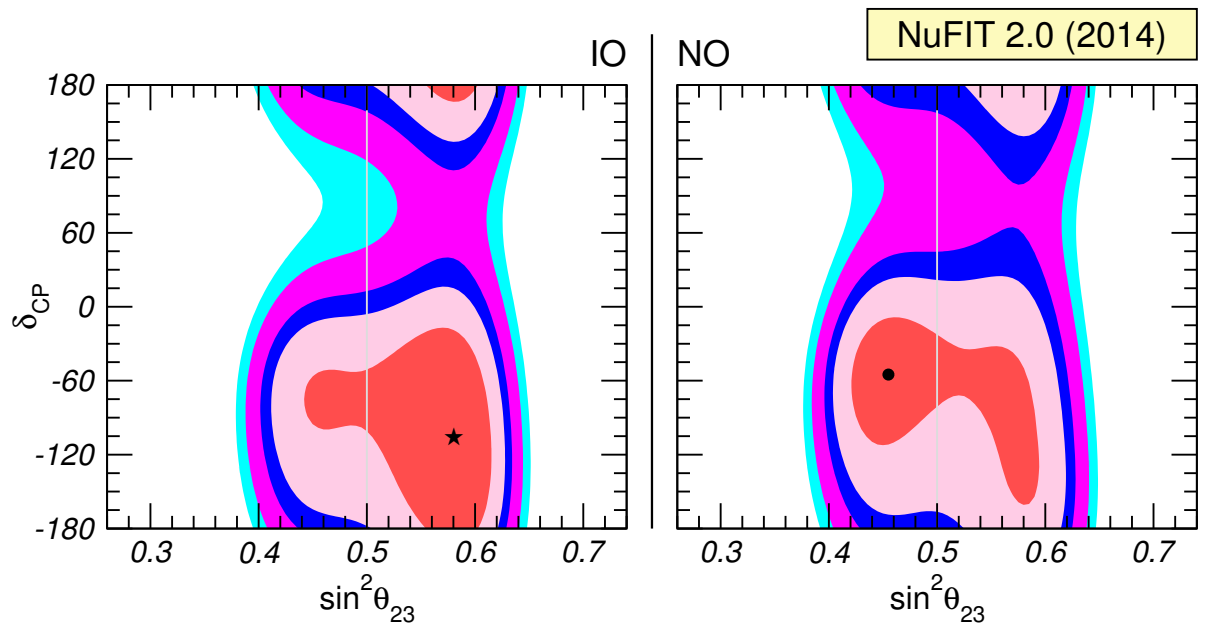

Figure 9. Allowed regions from the global data at $1 \sigma, 90 \%, 2 \sigma, 99 \%$ and $3 \sigma$ CL (2 dof) in the $\left(\theta_{23}, \delta_{\mathrm{CP}}\right)$ plane, after minimizing with respect to all undisplayed parameters. The left (right) panel corresponds to IO (NO). Contour regions in both panels are derived with respect to the global minimum which occurs for IO and is indicated by a star. The local minimum for NO is shown by a black dot.

In order to highlight the pattern of correlations between $\delta_{\mathrm{CP}}$ and $\sin ^{2} \theta_{23}$ we show in figure 9 the allowed regions of the global analysis projected into the plane of these two parameters. Correlations between $\delta_{\mathrm{CP}}$ and other oscillation parameters are mostly trivial and are therefore omitted.

\subsection{Remarks on confidence levels for $\delta_{\mathrm{CP}}$}

In order to study the information from data on the $\mathrm{CP}$ phase we consider the quantity

$$
\Delta \chi^{2}\left(\delta_{\mathrm{CP}}\right) \equiv \min _{x \neq \delta_{\mathrm{CP}}} \chi^{2}\left(\delta_{\mathrm{CP}}, x\right)-\chi_{\min }^{2}
$$

where the first term on the right hand side is minimized with respect to all oscillation parameters except $\delta_{\mathrm{CP}}\left(x=\theta_{12}, \theta_{13}, \theta_{23}, \Delta m_{21}^{2}, \Delta m_{31}^{2}\right)$ and the last term is the $\chi^{2}$ minimum with respect to all oscillation parameters. We have shown $\Delta \chi^{2}\left(\delta_{\mathrm{CP}}\right)$ for various data sets in the lower panels of figure 8 , as well as in the corresponding panel in figure 2 for the global data. The standard way to derive confidence intervals for $\delta_{\mathrm{CP}}$ is to assume that $\Delta \chi^{2}\left(\delta_{\mathrm{CP}}\right)$ follows a $\chi^{2}$-distribution with 1 dof, and then apply cuts corresponding to, e.g., $\Delta \chi^{2}=0.99,2.71,3.84,6.63$ for $68 \%, 90 \%, 95 \%, 99 \%$ CL, respectively. This procedure relies on Wilks theorem to hold [77]. However, in the case of $\delta_{\mathrm{CP}}$ some of the hypothesis of this theorem may be violated [78, 79]. One reason for this is the complicated nonlinear dependence of the event rates on $\delta_{\mathrm{CP}}$. Present sensitivity is so poor, that those non-linearities (as well as the periodic character of $\delta_{\mathrm{CP}}$ ) become relevant already at very

a detailed simulation of the detector, which can only be made by the experimental collaboration itself. Hence, although our results represent the most up-to-date analysis of the atmospheric neutrino data which can be performed outside the collaboration, such an analysis has unavoidable limitations. For details on our simulation of the data samples and the statistical analysis see the appendix of ref. [3]. 


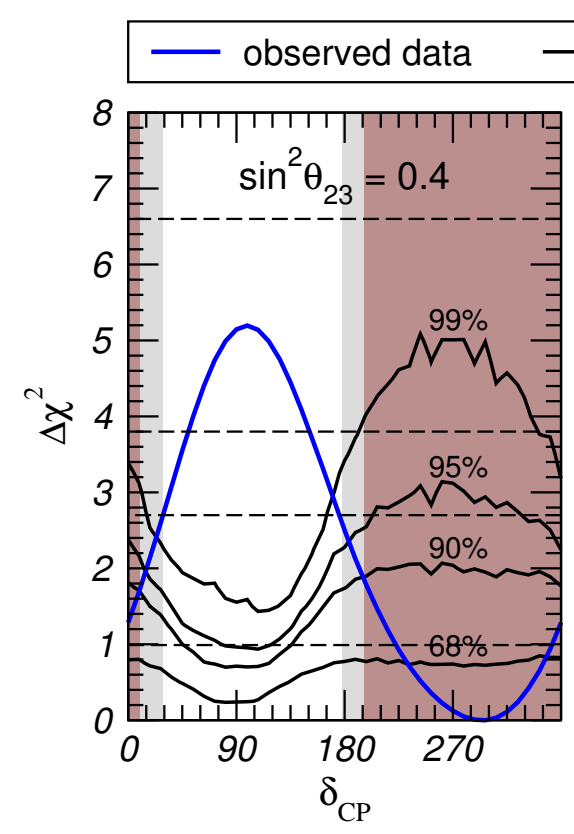

$\Delta \chi^{2}$ levels

NuFit 2.0 (2014)
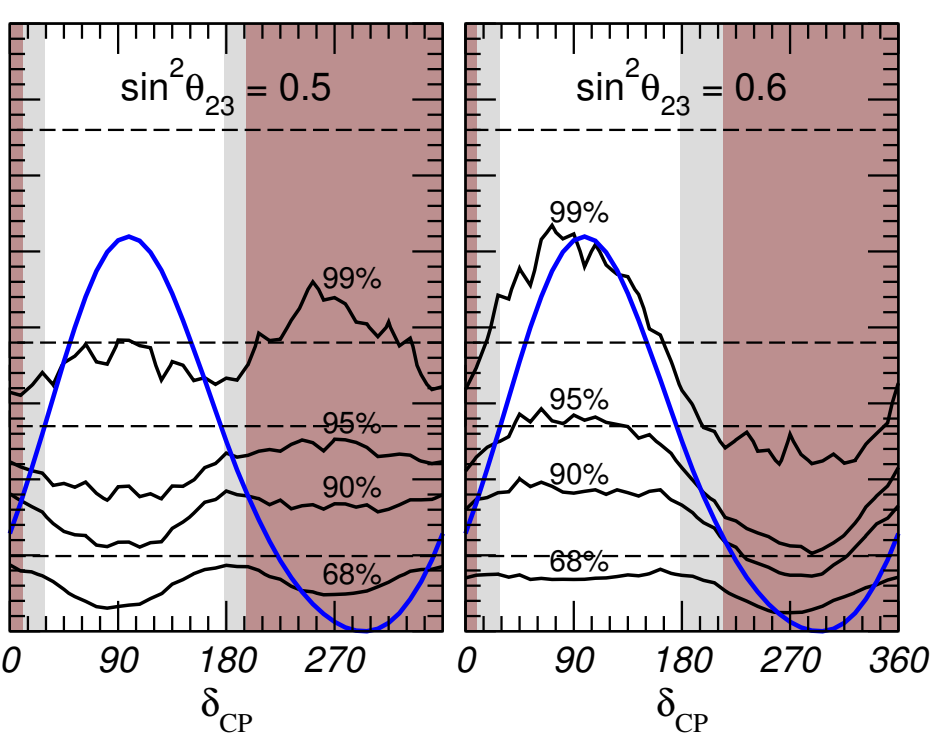

Figure 10. Black curves show the $\Delta \chi^{2}$ levels corresponding to $68 \%, 90 \%, 95 \%, 99 \%$ CL obtained from a Monte Carlo simulation of T2K appearance and disappearance data. Dashed lines correspond to the canonical values based on the $\chi^{2}$ distribution with 1 dof. The blue curve shows the observed $\Delta \chi^{2}$ using T2K data. The shaded regions indicate the $90 \%$ confidence interval for $\delta_{\mathrm{CP}}$ based on the distribution from simulated pseudo-data (brown) and on the $\chi^{2}$ approximation (gray). The three panels correspond to different assumptions on the true value of $\theta_{23}$ used to generate the pseudo-data. In the fit all parameters except $\delta_{\mathrm{CP}}$ and $\theta_{23}$ are fixed to the global best fit values, assuming normal mass ordering.

low CL. Furthermore, parameter degeneracies (especially with $\theta_{23}$, see discussion in the previous sub-section) affect the distribution of the test statistic $\Delta \chi^{2}$ from eq. (4.9).

In order to address such concerns we have performed a Monte Carlo study of T2K data (appearance and disappearance). We consider a test statistic similar to the $\Delta \chi^{2}$ given in eq. (4.9); however, in order to keep calculation time manageable we fix all oscillation parameters except $\delta_{\mathrm{CP}}$ and $\theta_{23}$ to their best fit values from the global fit assuming normal mass ordering. Hence, in the notation of eq. (4.9) we have only $x=\theta_{23}$. In particular, since we keep also $\theta_{13}$ fixed, the main feature of the complementarity of long-baseline appearance and medium-baseline reactor data is maintained. We have checked that allowing $\theta_{13}$ to vary imposing the constraint from Daya Bay data has a negligible impact on $\Delta \chi^{2}\left(\delta_{\mathrm{CP}}\right)$ compared to fixing it to the best fit value. The resulting $\Delta \chi^{2}\left(\delta_{\mathrm{CP}}\right)$ is shown as blue curve in figure 10 (identical in all three panels). It differs somewhat from the global result displayed in figures 2 or 8 , which include more data, but it captures the essential features and suffices for the purpose of studying the statistical properties of the test statistic.

In order to estimate the probability distribution for $\Delta \chi^{2}\left(\delta_{\mathrm{CP}}\right)$ we proceed as follows. We scan the parameter space of $\delta_{\mathrm{CP}}$ and $\sin ^{2} \theta_{23}$ and for a given point of assumed true values we generate a large number of pseudo-data samples for T2K. For each data set we calculate the value of the test statistic $\Delta \chi^{2}\left(\delta_{\mathrm{CP}}\right)$ and in this way we obtain a distribution 
for it. We scan 41 points in $\delta_{\mathrm{CP}}$ and 3 points for $\sin ^{2} \theta_{23}$, and for each of those points we generate 5000 pseudo-data samples. The black curves in figure 10 show the values of $\Delta \chi^{2}\left(\delta_{\mathrm{CP}}\right)$ which are larger than $68 \%, 90 \%, 95 \%$ and $99 \%$ of all generated data samples. We observe quite large deviations from the corresponding values based on the $\chi^{2}$-distribution for 1 dof, shown by the dashed lines in the figure. Interestingly we find also a rather strong dependence on the assumed true value of $\theta_{23}$.

The behavior of the curves can be understood qualitatively. Due to the non-linearity of $\delta_{\mathrm{CP}}$ (its cyclic nature) and the poor sensitivity mentioned above it actually counts as less than 1 full degree of freedom, which implies distributions more concentrated at lower values than the $\chi^{2}$-distribution for 1 dof, as observed in figure 10. The rather strong variations for non-maximal values of $\theta_{23}$, including a flipped behavior for $\delta_{\mathrm{CP}}$ smaller or larger $\pi$ between $\sin ^{2} \theta_{23}=0.4$ and 0.6 can be understood in terms of a degeneracy. For $\theta_{23}<\pi / 4$ and $\delta_{\mathrm{CP}} \sim 3 \pi / 2$ as well as for for $\theta_{23}>\pi / 4$ and $\delta_{\mathrm{CP}} \sim \pi / 2$ there is a degeneracy between the two octants of $\theta_{23}$ which effectively enhances the number of degrees of freedom in the fit. ${ }^{3}$

Now we can compare $\Delta \chi^{2}\left(\delta_{\mathrm{CP}}\right)$ obtained from the observed data to the expected distribution. If the observed $\Delta \chi^{2}\left(\delta_{\mathrm{CP}}\right)$ is larger than the values obtained for $x \%$ of the pseudo-data samples for that true value of $\delta_{\mathrm{CP}}$ we exclude this value of $\delta_{\mathrm{CP}}$ at the $x \%$ CL. In figure 10 we show as an example the resulting $90 \%$ confidence interval for $\delta_{\mathrm{CP}}$ as brown shaded area. This corresponds to the confidence interval according to the Feldman-Cousins (FC) prescription [80]. It has to be compared to the corresponding interval based on the $\chi^{2}$-approximation, indicated by the gray area in the plot.

We can draw the following conclusions from the exercise shown in figure 10:

1. the confidence intervals based on the Monte Carlo simulation are smaller than the ones based on the $\chi^{2}$-approximation. Hence, the latter is conservative;

2. for confidence levels $\lesssim 90 \%$ the confidence intervals are similar, whereas for higher confidence levels differences become significant. In particular, at 99\% CL all values of $\delta_{\mathrm{CP}}$ are allowed using the $\chi^{2}$-approximation, whereas a region around $\delta_{\mathrm{CP}} \sim \pi / 2$ remains excluded by the $99 \%$ CL FC interval;

3. the CL with which $\delta_{\mathrm{CP}} \sim \pi / 2$ can be disfavored depends strongly on the unknown true value of $\theta_{23}$. For $\sin ^{2} \theta_{23}=0.6, \delta_{\mathrm{CP}} \simeq \pi / 2$ is excluded at about $99 \% \mathrm{CL}$, whereas for $\sin ^{2} \theta_{23}=0.4$ it is excluded at very high CL. In all cases, the CL based on the Monte Carlo is higher than in the $\chi^{2}$-approximation which again can be considered conservative.

Let us conclude this section by commenting that ideally such a simulation should be performed also for the global analysis. Unfortunately this is currently out of question, in particular due to atmospheric neutrino data, which is very computational intensive and does play a non-negligible role in the global fit for $\Delta \chi^{2}\left(\delta_{\mathrm{CP}}\right)$, see figure 8 . However, we believe that the above results based on $\mathrm{T} 2 \mathrm{~K}$ are approximately representative also for the

\footnotetext{
${ }^{3}$ The presence of this degeneracy can be understood from eq. (4.6) considered at fixed $\theta_{13}$ and $\Delta \simeq \pi / 2$ (first oscillation maximum).
} 
global fit. One may expect that, with more statistics, distributions become more close to the expected $\chi^{2}$-distribution. However, preliminary estimates indicate that parameter degeneracies may lead to deviations also in a high-statistics scenario.

\section{Summary}

We have presented the results of an updated (as of summer 2014) global analysis of solar, atmospheric, reactor and accelerator neutrino data in the framework of three-neutrino oscillations. Quantitatively the present determination of the oscillation parameters is listed in table 1, and the corresponding leptonic mixing matrix is given in eq. (3.1). From the present analysis we have derived the maximum allowed $\mathrm{CP}$ violation in the leptonic sector as parametrized by the Jarlskog determinant, $J_{\mathrm{CP}}=0.033 \pm 0.010( \pm 0.027)$ at $1 \sigma(3 \sigma)$. All these results have also been shown in terms of unitarity triangles in figure 4 which further illustrate the ability of global oscillation data to obtain information on leptonic $\mathrm{CP}$ violation.

The global analysis presents a series of tensions between data sets as well as some 1-2 $\sigma$ effects in the determination of less known parameters $\left(\theta_{23}\right.$, mass ordering, and $\left.\delta_{\mathrm{CP}}\right)$ which we denote as "tendencies" and we discuss in section 4 . We can summarize these results as follows:

- due to the very precise determination of the flux-independent near-far ratio from Daya Bay and RENO, the so-called reactor neutrino anomaly (i.e., the tension between the predicted reactor fluxes in refs. $[45,46]$ and the event rates observed in short-baseline reactor experiments) results only in a $0.5 \sigma$ uncertainty on the determination of $\theta_{13}$;

- the long-standing $\sim 2 \sigma$ tension between the best fit values of $\Delta m_{21}^{2}$ as determined from the analysis of KamLAND and solar data is still unresolved. This tension is driven by both the indication of a non-zero day-night effect at SK, and by the lack of evidence of a low energy turn-up in the ${ }^{8} \mathrm{~B}$ energy spectrum as measured by SNO, SK4 and Borexino. In both cases the $\Delta m_{21}^{2}$ value favored by KamLAND is in disagreement with the expectations from the standard LMA-MSW solution;

- the uncertainty on the determination of $\Delta m_{21}^{2}$ and $\theta_{12}$ due to the choice of Standard Solar Model associated with the "solar composition problem" is negligible;

- at present the precision on the determination of $\left|\Delta m_{3 \ell}^{2}\right|$ from $\nu_{\mu}$ disappearance experiments (mainly T2K and MINOS) is comparable to that from $\nu_{e}$ disappearance experiments (i.e. reactor experiments including, in particular, the spectral information from Daya Bay);

- for Inverted Ordering, the "tendency" towards non-maximal mixing and second octant of $\theta_{23}$ is driven mainly by two effects: (a) the non-maximality favored by MINOS $\nu_{\mu}$ disappearance, and (b) the "mismatch" between the best fit $\theta_{13}$ obtained from $\bar{\nu}_{e}$ disappearance at reactors and from $\nu_{\mu} \rightarrow \nu_{e}$ at T2K. Atmospheric results do not alter this; 
- for Normal Ordering, such preference for non-maximal $\theta_{23}$ mixing is considerably weaker than for IO; also, in this case the global best-fit occur in the first $\theta_{23}$ octant, mostly driven by atmospheric data;

- the "mismatch" between reactor and T2K results is the driving effect in the present dependence of the global $\Delta \chi^{2}$ on the $\mathrm{CP}$ violating phase with a best fit value close to $\delta_{\mathrm{CP}}=\frac{3}{2} \pi$. Inclusion of the atmospheric results adds positively to this effect for both orderings;

- the tendency towards IO or NO in the present analysis does not seem to result from any consistent effect and it shifts in sign depending on the data sets considered.

Finally in section 4.5 we have addressed the issue of the "gaussianity" of the confidence levels attributed to $\Delta \chi^{2}\left(\delta_{\mathrm{CP}}\right)$ by performing a Monte Carlo study of T2K data, and we have compared the resulting probability distribution to that of a $\chi^{2}$-distribution as usually assumed. Deviations are expected due to the cyclic nature of $\delta_{\mathrm{CP}}$ and to the presence of parameter degeneracies. The conclusion is that, within the present data, the use of the $\chi^{2}$-distribution approximation is slightly conservative in the determination of the excluded range of $\delta_{\mathrm{CP} P}$ at confidence levels $\gtrsim 90 \%$. The differences however are not very significant as illustrated in figure 10 .

Future updates of this analysis will be provided at the website quoted in ref. [12].

\section{Acknowledgments}

We would like to thank M. Smy for pointing out to us the importance of the day-night asymmetry for the tension between the solar and KamLAND fits. T.S. also thanks Mattias Blennow, Pilar Coloma and Enrique Fernandez-Martinez for extensive discussions on the results presented in section 4.5. This work is supported by Spanish MINECO grants FPA2012-31880, FPA2012-34694 and FPA2013-46570, by the Severo Ochoa program SEV-2012-0249 and consolider-ingenio 2010 grant CSD-2008-0037, by CUR Generalitat de Catalunya grant 2009SGR502, by USA-NSF grant PHY-09-69739 and PHY-13-16617, and by EU grant FP7 ITN INVISIBLES (Marie Curie Actions PITN-GA-2011-289442).

\section{A List of data used in the analysis}

\section{Solar experiments}

- Chlorine total rate [19], 1 data point.

- Gallex \& GNO total rates [20], 2 data points.

- SAGE total rate [21], 1 data point.

- SK1 full energy and zenith spectrum [22], 44 data points.

- SK2 full energy and day/night spectrum [23], 33 data points. 
- SK3 full energy and day/night spectrum [24], 42 data points.

- SK4 1669-day energy spectrum and day/night asymmetry [25], 24 data points.

- SNO combined analysis [26], 7 data points.

- Borexino 740.7-day low-energy data [27], 33 data points.

- Borexino 246-day high-energy data [28], 6 data points.

\section{Atmospheric experiments}

- SK1-4 (including SK4 1775-day) combined data [13], 70 data points.

\section{Reactor experiments}

- KamLAND combined DS1 \& DS2 spectrum [35], 17 data points.

- CHOOZ energy spectrum [30], 14 data points.

- Palo Verde total rate [31], 1 data point.

- Double Chooz 227.9-day spectrum [32], 18 data points.

- Daya Bay 621-day spectrum [33], 36 data points.

- RENO 800-day near \& far total rates [34], 2 data points (with free normalization).

- SBL reactor data (including Daya-Bay total flux at near detector), 77 data points [33, 36-44].

\section{Accelerator experiments}

- MINOS $10.71 \times 10^{20}$ pot $\nu_{\mu}$-disappearance data [15], 39 data points.

- MINOS $3.36 \times 10^{20}$ pot $\bar{\nu}_{\mu}$-disappearance data [15], 14 data points.

- MINOS $10.6 \times 10^{20}$ pot $\nu_{e}$-appearance data [17], 5 data points.

- MINOS $3.3 \times 10^{20}$ pot $\bar{\nu}_{e}$-appearance data [17], 5 data points.

- T2K $6.57 \times 10^{20}$ pot $\nu_{\mu}$-disappearance data [16], 16 data points.

- $\mathrm{T} 2 \mathrm{~K} 6.57 \times 10^{20}$ pot $\nu_{e}$-appearance data [18], 5 data points.

Open Access. This article is distributed under the terms of the Creative Commons Attribution License (CC-BY 4.0), which permits any use, distribution and reproduction in any medium, provided the original author(s) and source are credited. 


\section{References}

[1] B. Pontecorvo, Neutrino experiments and the problem of conservation of leptonic charge, Sov. Phys. JETP 26 (1968) 984 [Zh. Eksp. Teor. Fiz. 53 (1967) 1717] [InSPIRE].

[2] V.N. Gribov and B. Pontecorvo, Neutrino astronomy and lepton charge, Phys. Lett. B 28 (1969) 493 [INSPIRE].

[3] M.C. Gonzalez-Garcia and M. Maltoni, Phenomenology with massive neutrinos, Phys. Rept. 460 (2008) 1 [arXiv:0704.1800] [INSPIRE].

[4] J. Kopp, P.A.N. Machado, M. Maltoni and T. Schwetz, Sterile neutrino oscillations: the global picture, JHEP 05 (2013) 050 [arXiv: 1303.3011] [INSPIRE].

[5] Z. Maki, M. Nakagawa and S. Sakata, Remarks on the unified model of elementary particles, Prog. Theor. Phys. 28 (1962) 870 [InSPIRE].

[6] M. Kobayashi and T. Maskawa, CP violation in the renormalizable theory of weak interaction, Prog. Theor. Phys. 49 (1973) 652 [InSPIRE].

[7] Particle Data Group collaboration, J. Beringer et al., Review of particle physics (RPP), Phys. Rev. D 86 (2012) 010001 [INSPIRE].

[8] S.M. Bilenky, J. Hosek and S.T. Petcov, On oscillations of neutrinos with Dirac and Majorana masses, Phys. Lett. B 94 (1980) 495 [InSPIRE].

[9] P. Langacker, S.T. Petcov, G. Steigman and S. Toshev, On the Mikheev-Smirnov-Wolfenstein (MSW) mechanism of amplification of neutrino oscillations in matter, Nucl. Phys. B 282 (1987) 589 [INSPIRE].

[10] F. Capozzi et al., Status of three-neutrino oscillation parameters, circa 2013, Phys. Rev. D 89 (2014) 093018 [arXiv:1312.2878] [INSPIRE].

[11] D.V. Forero, M. Tortola and J.W.F. Valle, Neutrino oscillations refitted, arXiv:1405.7540 [INSPIRE].

[12] NuFIT webpage, http://www.nu-fit.org/.

[13] R. Wendell, Atmospheric results from Super-Kamiokande, talk given at the XXVI International Conference on Neutrino Physics and Astrophysics, Boston U.S.A. June 2-7 2014.

[14] Super-Kamiokande collaboration, R. Wendell et al., Atmospheric neutrino oscillation analysis with sub-leading effects in Super-Kamiokande I, II and III, Phys. Rev. D 81 (2010) 092004 [arXiv: 1002.3471] [INSPIRE].

[15] MINOS collaboration, P. Adamson et al., Measurement of neutrino and antineutrino oscillations using beam and atmospheric data in MINOS, Phys. Rev. Lett. 110 (2013) 251801 [arXiv: 1304.6335] [INSPIRE].

[16] T2K collaboration, K. Abe et al., Precise measurement of the neutrino mixing parameter $\theta_{23}$ from muon neutrino disappearance in an off-axis beam, Phys. Rev. Lett. 112 (2014) 181801 [arXiv: 1403.1532] [INSPIRE].

[17] MINOS collaboration, P. Adamson et al., Electron neutrino and antineutrino appearance in the full MINOS data sample, Phys. Rev. Lett. 110 (2013) 171801 [arXiv:1301.4581] [INSPIRE]. 
[18] T2K collaboration, K. Abe et al., Observation of electron neutrino appearance in a muon neutrino beam, Phys. Rev. Lett. 112 (2014) 061802 [arXiv:1311.4750] [INSPIRE].

[19] B.T. Cleveland et al., Measurement of the solar electron neutrino flux with the Homestake chlorine detector, Astrophys. J. 496 (1998) 505 [INSPIRE].

[20] F. Kaether, W. Hampel, G. Heusser, J. Kiko and T. Kirsten, Reanalysis of the GALLEX solar neutrino flux and source experiments, Phys. Lett. B 685 (2010) 47 [arXiv:1001.2731] [INSPIRE].

[21] SAGE collaboration, J.N. Abdurashitov et al., Measurement of the solar neutrino capture rate with gallium metal. III: results for the 2002-2007 data-taking period, Phys. Rev. C 80 (2009) 015807 [arXiv:0901.2200] [INSPIRE].

[22] Super-Kamiokande collaboration, J. Hosaka et al., Solar neutrino measurements in Super-Kamiokande-I, Phys. Rev. D 73 (2006) 112001 [hep-ex/0508053] [InSPIRE].

[23] Super-Kamiokande collaboration, J.P. Cravens et al., Solar neutrino measurements in Super-Kamiokande-II, Phys. Rev. D 78 (2008) 032002 [arXiv:0803.4312] [InSPIRE].

[24] Super-Kamiokande collaboration, K. Abe et al., Solar neutrino results in Super-Kamiokande-III, Phys. Rev. D 83 (2011) 052010 [arXiv:1010.0118] [InSPIRE].

[25] Y. Koshio, Solar results from Super-Kamiokande, talk given at the XXVI International Conference on Neutrino Physics and Astrophysics, Boston U.S.A. June 2-7 2014.

[26] SNO collaboration, B. Aharmim et al., Combined analysis of all three phases of solar neutrino data from the Sudbury Neutrino Observatory, Phys. Rev. C 88 (2013) 025501 [arXiv:1109.0763] [INSPIRE].

[27] Borexino collaboration, G. Bellini et al., Precision measurement of the ${ }^{7}$ Be solar neutrino interaction rate in Borexino, Phys. Rev. Lett. 107 (2011) 141302 [arXiv:1104.1816] [INSPIRE].

[28] Borexino collaboration, G. Bellini et al., Measurement of the solar ${ }^{8} B$ neutrino rate with a liquid scintillator target and $3 \mathrm{MeV}$ energy threshold in the Borexino detector, Phys. Rev. D 82 (2010) 033006 [arXiv: 0808.2868] [INSPIRE].

[29] A. Serenelli, S. Basu, J.W. Ferguson and M. Asplund, New solar composition: the problem with solar models revisited, Astrophys. J. 705 (2009) L123 [arXiv:0909.2668] [INSPIRE].

[30] CHOOZ collaboration, M. Apollonio et al., Limits on neutrino oscillations from the CHOOZ experiment, Phys. Lett. B 466 (1999) 415 [hep-ex/9907037] [INSPIRE].

[31] PALo Verde collaboration, A. Piepke, Final results from the Palo Verde neutrino oscillation experiment, Prog. Part. Nucl. Phys. 48 (2002) 113 [InSPIRE].

[32] Double CHOOZ collaboration, Y. Abe et al., Reactor electron antineutrino disappearance in the Double CHOOZ experiment, Phys. Rev. D 86 (2012) 052008 [arXiv:1207.6632] [INSPIRE].

[33] C. Zhang, Recent results from Daya Bay, talk given at the XXVI International Conference on Neutrino Physics and Astrophysics, Boston U.S.A. June 2-7 2014.

[34] S.-H. Seo, New results from RENO, talk given at the XXVI International Conference on Neutrino Physics and Astrophysics, Boston U.S.A. June 2-7 2014. 
[35] KamLAND collaboration, A. Gando et al., Constraints on $\theta_{13}$ from a three-flavor oscillation analysis of reactor antineutrinos at KamLAND, Phys. Rev. D 83 (2011) 052002 [arXiv: 1009.4771] [INSPIRE].

[36] Y. Declais et al., Study of reactor anti-neutrino interaction with proton at Bugey nuclear power plant, Phys. Lett. B 338 (1994) 383 [INSPIRE].

[37] A.A. Kuvshinnikov, L.A. Mikaelyan, S.V. Nikolaev, M.D. Skorokhvatov and A.V. Etenko, Measuring the $\bar{\nu}_{e}+p \rightarrow n+e^{+}$cross-section and beta decay axial constant in a new experiment at Rovno NPP reactor (in Russian), JETP Lett. 54 (1991) 253 [Yad. Fiz. 52 (1990) 472] [Pisma Zh. Eksp. Teor. Fiz. 54 (1991) 259] [Sov. J. Nucl. Phys. 52 (1990) 300] [INSPIRE].

[38] Y. Declais et al., Search for neutrino oscillations at 15-meters, 40-meters and 95-meters from a nuclear power reactor at Bugey, Nucl. Phys. B 434 (1995) 503 [inSPIRE].

[39] G.S. Vidyakin et al., Detection of anti-neutrinos in the flux from two reactors, Sov. Phys. JETP 66 (1987) 243 [Zh. Eksp. Teor. Fiz. 93 (1987) 424] [inSPIRE].

[40] G.S. Vidyakin et al., Limitations on the characteristics of neutrino oscillations, JETP Lett. 59 (1994) 390 [Pisma Zh. Eksp. Teor. Fiz. 59 (1994) 364] [InSPIRE].

[41] H. Kwon et al., Search for neutrino oscillations at a fission reactor, Phys. Rev. D 24 (1981) 1097 [INSPIRE].

[42] CALTECH-SIN-TUM collaboration, G. Zacek et al., Neutrino oscillation experiments at the Gosgen nuclear power reactor, Phys. Rev. D 34 (1986) 2621 [INSPIRE].

[43] Z.D. Greenwood et al., Results of a two position reactor neutrino oscillation experiment, Phys. Rev. D 53 (1996) 6054 [INSPIRE].

[44] A.I. Afonin et al., A study of the reaction $\bar{\nu}_{e}+p \rightarrow e^{+}+n$ on a nuclear reactor, Sov. Phys. JETP 67 (1988) 213 [Zh. Eksp. Teor. Fiz. 94N2 (1988) 1] [INSPIRE].

[45] P. Huber, On the determination of anti-neutrino spectra from nuclear reactors, Phys. Rev. C 84 (2011) 024617 [Erratum ibid. C 85 (2012) 029901] [arXiv: 1106.0687] [INSPIRE].

[46] T. Mueller et al., Improved predictions of reactor antineutrino spectra, Phys. Rev. C 83 (2011) 054615 [arXiv:1101.2663] [INSPIRE].

[47] G. Mention et al., The reactor antineutrino anomaly, Phys. Rev. D 83 (2011) 073006 [arXiv:1101.2755] [INSPIRE].

[48] T. Schwetz, M. Tortola and J.W.F. Valle, Global neutrino data and recent reactor fluxes: status of three-flavour oscillation parameters, New J. Phys. 13 (2011) 063004 [arXiv: 1103.0734] [INSPIRE].

[49] M.C. Gonzalez-Garcia, M. Maltoni, J. Salvado and T. Schwetz, Global fit to three neutrino mixing: critical look at present precision, JHEP 12 (2012) 123 [arXiv: 1209.3023] [INSPIRE].

[50] M.C. Gonzalez-Garcia and C. Pena-Garay, Three neutrino mixing after the first results from K2K and KamLAND, Phys. Rev. D 68 (2003) 093003 [hep-ph/0306001] [INSPIRE].

[51] C. Jarlskog, Commutator of the quark mass matrices in the standard electroweak model and a measure of maximal CP-violation, Phys. Rev. Lett. 55 (1985) 1039 [INSPIRE].

[52] Y. Farzan and A.Y. Smirnov, Leptonic unitarity triangle and CP-violation, Phys. Rev. D 65 (2002) 113001 [hep-ph/0201105] [INSPIRE]. 
[53] A.Y. Smirnov, Neutrino-2008: where are we? Where are we going?, J. Phys. Conf. Ser. 136 (2008) 012002 [arXiv:0810.2668] [INSPIRE].

[54] A. Dueck, S. Petcov and W. Rodejohann, On leptonic unitary triangles and boomerangs, Phys. Rev. D 82 (2010) 013005 [arXiv: 1006.0227] [InSPIRE].

[55] H.-J. He and X.-J. Xu, Connecting leptonic unitarity triangle to neutrino oscillation, Phys. Rev. D 89 (2014) 073002 [arXiv:1311.4496] [InSPIRE].

[56] S. Goswami and A.Y. Smirnov, Solar neutrinos and 1-3 leptonic mixing, Phys. Rev. D 72 (2005) 053011 [hep-ph/0411359] [INSPIRE].

[57] L. Wolfenstein, Neutrino oscillations in matter, Phys. Rev. D 17 (1978) 2369 [INSPIRE].

[58] S.P. Mikheev and A.Y. Smirnov, Resonance amplification of oscillations in matter and spectroscopy of solar neutrinos, Sov. J. Nucl. Phys. 42 (1985) 913 [Yad. Fiz. 42 (1985) 1441] [INSPIRE].

[59] G.L. Fogli, E. Lisi, A. Marrone, A. Palazzo and A.M. Rotunno, Hints of $\theta_{13}>0$ from global neutrino data analysis, Phys. Rev. Lett. 101 (2008) 141801 [arXiv:0806.2649] [INSPIRE].

[60] G.L. Fogli, E. Lisi, A. Marrone, A. Palazzo and A.M. Rotunno, Neutrino masses and mixing: 2008 status, Nucl. Phys. Proc. Suppl. 188 (2009) 27 [InSPIRE].

[61] T. Schwetz, M.A. Tortola and J.W.F. Valle, Three-flavour neutrino oscillation update, New J. Phys. 10 (2008) 113011 [arXiv:0808. 2016] [INSPIRE].

[62] M. Maltoni and T. Schwetz, Three-flavour neutrino oscillation update and comments on possible hints for a non-zero $\theta_{13}$, PoS (IDM2008) 072 [arXiv:0812.3161] [INSPIRE].

[63] A.B. Balantekin and D. Yilmaz, Contrasting solar and reactor neutrinos with a non-zero value of $\theta_{13}$, J. Phys. G 35 (2008) 075007 [arXiv:0804.3345] [InSPIRE].

[64] M.C. Gonzalez-Garcia, M. Maltoni and J. Salvado, Updated global fit to three neutrino mixing: status of the hints of $\theta_{13}>0$, JHEP 04 (2010) 056 [arXiv: 1001.4524] [INSPIRE].

[65] A. Palazzo, Hint of non-standard dynamics in solar neutrino conversion, Phys. Rev. D 83 (2011) 101701 [arXiv:1101.3875] [INSPIRE].

[66] M.C. Gonzalez-Garcia and M. Maltoni, Determination of matter potential from global analysis of neutrino oscillation data, JHEP 09 (2013) 152 [arXiv: 1307.3092] [INSPIRE].

[67] P.C. de Holanda and A.Y. Smirnov, Solar neutrino spectrum, sterile neutrinos and additional radiation in the universe, Phys. Rev. D 83 (2011) 113011 [arXiv:1012.5627] [INSPIRE].

[68] T.J.C. Bezerra, H. Furuta and F. Suekane, Measurement of effective $\Delta m_{31}^{2}$ using baseline differences of Daya Bay, RENO and Double CHOOZ reactor neutrino experiments, arXiv: 1206.6017 [INSPIRE].

[69] Daya BAY collaboration, F.P. An et al., Spectral measurement of electron antineutrino oscillation amplitude and frequency at Daya Bay, Phys. Rev. Lett. 112 (2014) 061801 [arXiv: 1310.6732] [INSPIRE].

[70] A. Cervera et al., Golden measurements at a neutrino factory, Nucl. Phys. B 579 (2000) 17 [Erratum ibid. B 593 (2001) 731] [hep-ph/0002108] [INSPIRE].

[71] M. Freund, Analytic approximations for three neutrino oscillation parameters and probabilities in matter, Phys. Rev. D 64 (2001) 053003 [hep-ph/0103300] [INSPIRE]. 
[72] E.K. Akhmedov, R. Johansson, M. Lindner, T. Ohlsson and T. Schwetz, Series expansions for three flavor neutrino oscillation probabilities in matter, JHEP 04 (2004) 078 [hep-ph/0402175] [INSPIRE].

[73] C.W. Kim and U.W. Lee, Comment on the possible electron neutrino excess in the Super-Kamiokande atmospheric neutrino experiment, Phys. Lett. B 444 (1998) 204 [hep-ph/9809491] [INSPIRE].

[74] O.L.G. Peres and A.Y. Smirnov, Testing the solar neutrino conversion with atmospheric neutrinos, Phys. Lett. B 456 (1999) 204 [hep-ph/9902312] [INSPIRE].

[75] O.L.G. Peres and A.Y. Smirnov, Atmospheric neutrinos: LMA oscillations, $U_{e 3}$ induced interference and CP-violation, Nucl. Phys. B 680 (2004) 479 [hep-ph/0309312] [INSPIRE].

[76] M.C. Gonzalez-Garcia, M. Maltoni and A.Y. Smirnov, Measuring the deviation of the 2-3 lepton mixing from maximal with atmospheric neutrinos, Phys. Rev. D 70 (2004) 093005 [hep-ph/0408170] [INSPIRE].

[77] S.S. Wilks, The large-sample distribution of the likelihood ratio for testing composite hypotheses, Annals Math. Statist. 9 (1938) 60 [INSPIRE].

[78] T. Schwetz, What is the probability that $\theta_{13}$ and CP-violation will be discovered in future neutrino oscillation experiments?, Phys. Lett. B 648 (2007) 54 [hep-ph/0612223] [INSPIRE].

[79] M. Blennow, P. Coloma and E. Fernandez-Martinez, Reassessing the sensitivity to leptonic CP-violation, arXiv:1407.3274 [INSPIRE].

[80] G.J. Feldman and R.D. Cousins, A unified approach to the classical statistical analysis of small signals, Phys. Rev. D 57 (1998) 3873 [physics/9711021] [INSPIRE]. 